\title{
OPERATION OF A PERFUSIVE CATALYTIC MEMBRANE WITH NONLINEAR KINETICS
}

\author{
J. P. Lopes ${ }^{1, *}$, M. A. Alves ${ }^{2}$, M. S. N. Oliveira ${ }^{3}$, S. S. S. Cardoso ${ }^{4}$ and A. E. Rodrigues ${ }^{1}$ \\ ${ }^{1}$ Laboratory of Separation and Reaction Engineering, Associate Laboratory LSRE/LCM, University of Porto, Rua \\ Dr. Roberto Frias s/n, 4200-465 Porto, Portugal \\ ${ }^{2}$ Transport Phenomena Research Center (CEFT), Department of Chemical Engineering, University of Porto, Rua Dr. \\ Roberto Frias s/n, 4200-465 Porto, Portugal \\ ${ }^{3}$ James Weir Fluids Lab, Department of Mechanical and Aerospace Engineering, University of Strathclyde, Glasgow \\ G1 1XJ, UK \\ ${ }^{4}$ Department of Chemical Engineering and Biotechnology, University of Cambridge, New Museums Site, Pembroke \\ Street, Cambridge, CB2 3RA, UK \\ *Corresponding author. J. P. Lopes. Tel.: +351 22508 1578. E-mail: jplopes@fe.up.pt
}

\section{AbSTRACT}

Operation of a perfusive catalytic curved membrane is systematized into different transportreaction regimes. The internal viscous permeation improves the catalyst performance, measured here by the effectiveness factor and by its enhancement relative to purely diffusive conditions. A theoretical analysis is presented for nonlinear kinetic expressions, which are suitable to describe the consumption of a reactant in many (bio)catalytic systems. The kinetic and transport parameters required to attain maximum enhancement are related by simple design rules, which depend on the form of the reaction rate law (namely on the order of reaction and dimensionless inhibition constant). For zero-order reactions, these optimum conditions correspond to attaining negligible concentration at a position inside the membrane, while generically may be interpreted as separating situations of severe mass transfer resistance from cases of high effectiveness. It is important to incorporate the correct kinetic expression in the analysis, so that the predictions can be used in a quantitative manner. The results for the different regimes are compiled in enhancement plots and in Peclet-Thiele diagrams. Moreover, the study also yielded new results for the nonlinear reaction-diffusion in a curved membrane with its two surfaces exposed to different concentrations, a case of relevance in membrane reactors. 


\section{KEYWORDS}

Membrane; Effectiveness factor; Michaelis-Menten; Viscous flow; Perfusive bioreactor

\section{INTRODUCTION}

Owing to the slow nature of diffusion in conventional porous catalysts, permeable catalytic materials have proven to be of practical interest since promoting an internal flow field increases the mass transport rate. This concept has been investigated both theoretically $[1,2]$ and experimentally [3-5] in a broad range of conditions. It is not only found in reaction engineering $[6,7]$, but also in separation processes (as chromatographic supports $[8,9]$ ), or in the fields of biotechnology (as supports for cell culture and biomass growth [10-14]), among others. In some sense, it is also related with materials showing a hierarchy of pore sizes [15] including the macropore range.

Reactor designs based on these materials have been presented as monoliths $[16,17]$, porous ceramic mesoreactors (e.g. for multiphase hydrogenations [18-20]), flow-through filters [21, 22], coated microchannels with carbon nanofiber layers [23] or as hollow fiber bio- and enzymatic reactors $[12,24,25]$. They are also frequently classified as non-permselective catalytic membrane reactors [26-36]. Higher catalyst activity (and in some cases, higher selectivity) were observed due to the intensification in the transport of reactants by convection in the selective epoxidation of propene [34], catalytic reduction of nitrite in water and dechlorination of chloroform [37], and the catalytic oxidation of propene [38]. The same concept was applied to the partial hydrogenation of 1,5-cyclooctadiene [39], $\alpha$-methylstyrene hydrogenation [40] and nitrate reduction [41] in a similar design. Earlier work on non-selective membranes had also the objective of controlling the rates of separate feeding of different reactants [42, 43]. More recently, Murru and Gavriilidis [44] studied the catalytic combustion of methane with the same configuration in open and dead-end modes. The application of a pressure difference, inducing a convective contribution to the radial fluxes, improved the performance by decreasing the concentrations of carbon monoxide and hydrogen.

These materials have also been integrated in microfluidic catalytic reactors [45] and cell culture devices [46] to model physiological phenomena. Hsu et al. [47] proposed a microfluidic 
platform to study mass transport in tissue cultures. In particular, the ratio between the timescales for diffusion and convection (compared in the Peclet number) was varied by 5 orders of magnitude (values up to 160). They studied the influence of hypoxic conditions in diffusion limited systems (Peclet numbers below 0.1) and interstitial flow (significant when Peclet number is above 10) on vasculogenesis. Moreover, some microchips are designed to mimic bipolar cell environments (surfaces exposed at different conditions) and to study the response of cells to diffusive or convective-dominated delivery of certain agents [48].

Frequently, the description of these processes involves a nonlinear reaction rate law. Firstorder kinetics can provide not only exact solutions, but also convenient approximations [49]. However, biocatalytic perfusive reactors usually exhibit non-negligible levels of reactant inhibition. This added complexity leads to a major distinction in the theoretical treatment of the problem, since solutions valid for all values of the reaction rate cannot be obtained. Therefore, it is necessary to break down the analysis into the relevant reaction-transport regimes. Kinetically controlled conditions assume that reactant transport (which may be dominated by diffusion, convection or both) is much faster than the rate at which it is consumed by reaction. On the other hand, if the reaction is fast, two distinct situations are of interest: ( $i$ ) the diffusive fluxes near the surfaces are large, while convective transport is negligible (diffusional regime), or (ii) convection is comparably strong throughout the whole membrane thickness (convection dominated regime). We will show that considering separate regimes (with solutions of restricted validity in each one) aids the understanding and description of the full picture, and kinetic normalization is achieved. We also demonstrate how scaling, asymptotic and other approximate techniques can be employed in this case. Moreover, new results for purely diffusive catalytic membranes are presented, which do not seem to have been sufficiently considered in this context.

In section 2, a simple mass transfer model, which is able to capture the main features in perfusive membrane reactors, is presented. Then, several operating regimes are characterized, and the transitions between them identified (sections 3 to 5). Finally, the range of validity of these limiting behaviors is shown in a parametric map, illustrating the overall picture of the system (section 6). 


\section{CONVECTION, DIFFUSION AND NONLINEAR REACTION}

The operation of the catalytic membrane is determined by the rates of reactant transport (by convection and diffusion) and consumption, which must vary so that conservation of mass is satisfied:

$$
\frac{\partial^{2} c}{\partial r^{2}}+\frac{\varepsilon}{1+\varepsilon r} \frac{\partial c}{\partial r}-\frac{P}{1+\varepsilon r} \frac{\partial c}{\partial r}=\frac{\partial^{2} c}{\partial r^{2}}-\frac{P_{C}}{1+\varepsilon r} \frac{\partial c}{\partial r}=\phi^{2} R(c)
$$

As shown in Fig. 1, the boundary conditions for Eq.(1) may be those of specified surface concentration, $c(r=0)=1$ and $c(r=1)=c_{2}$ (if the dimensional concentration $\hat{c}$ is normalized by the value at the inner surface $\hat{c}_{1}$, as $c=\hat{c} / \hat{c}_{1}$ ), or of no-flux type at the downstream surface, if the membrane operates exclusively in radial flow model $[50,51]$. The former case may be appropriate to describe axial flow through the lumen and/or in the space surrounding the membrane, while the latter concerns the case where transmembrane flow dominates. Both conditions are important (see Fig. 2), since these membranes can work as contactors between different streams carrying the reactant, in the presence or absence of a sweep phase, with relatively weaker or stronger radial flow.

In the left-hand side of Eq.(1), the diffusive term was separated to show the contributions from planar diffusion and curvature. The dimensionless parameters that compare the timescale for diffusion in a slab-shaped membrane with the effects of convection and curvature are respectively: the Peclet number ( $P=V\langle\hat{u}\rangle /\left(S D_{\text {eff }}\right)$, positive for outward flow) and the ratio of length scales $\left(\varepsilon=t_{m} / a\right.$, see Fig. 1). Both dimensionless numbers can be combined into $P_{C}=P-\varepsilon$, as shown in (1). The average superficial velocity $\langle\hat{u}\rangle$ can be estimated by Darcy's law as a function of the membrane permeability and transmembrane pressure difference. Axial diffusion is neglected $\left(t_{m}<<L\right.$ and negligible permeability in this direction). The axial dependence of the solution is thus given by the parameter $P(\hat{z})$ and boundary condition $c_{2}(\hat{z})$. Note that for inward flow (permeate collected in the lumen of the channel), $P<0$.

In general, the empirical laws for the rate of several reactions are nonlinear and may be expressed as 
$R(c)=\left(\frac{1+k_{i}}{1+k_{i} c}\right)^{p} c^{m}$

or $R(c)=c^{m}$ for power-law kinetics $\left(k_{i}\right.$ is the inhibition rate constant multiplied by the scale for concentration, $k_{i}=k_{i}^{*} \hat{c}_{1}$ with $k_{i}^{*}$ in $\left.\mathrm{m}^{3} / \mathrm{mol}\right)$. Here, $R(c)$ is the dimensionless reaction rate, normalized by $\hat{R}\left(\hat{c}_{1}\right)=k \hat{c}_{1}^{m} /\left(1+k_{i}^{*} \hat{c}_{1}\right)^{p}$ in the Thiele modulus:

$\phi^{2}=\frac{t_{m}^{2}}{D_{\text {eff }}} \frac{\hat{R}\left(\hat{c}_{1}\right)}{\hat{c}_{1}}=\frac{t_{m}^{2}}{D_{\text {eff }}} \frac{k \hat{c}_{1}^{m-1}}{\left(1+k_{i}\right)^{p}}$.

The Michaelis-Menten kinetics is recovered when setting $m=p=1$. Even though the detailed concentration profile inside the membrane is of interest, more insightful (qualitative and quantitative) information is achieved by considering the averaged reaction rate over the whole membrane cross-section, i.e. the effectiveness factor,

$\eta=\frac{1}{v} \int_{0}^{1}(1+\varepsilon r) R(c) d r=\frac{1}{\phi^{2} v}\left[\left.(1+\varepsilon) \frac{\partial c}{\partial r}\right|_{r=1}-\left.\frac{\partial c}{\partial r}\right|_{r=0}\right]+[c(r=0)-c(r=1)] \frac{P}{\phi^{2} v}$.

Note that the ratio between the membrane volume $(V)$, its thickness $\left(t_{m}\right)$ and the area of the internal surface $(S)$ is given by $v=V /\left(t_{m} S\right)$, which is equal to $v=1$ or $v=1+\varepsilon / 2$, for planar or cylindrical membranes, respectively. A substantial part of the analysis presented in this work is concerned with the determination of analytical expressions for $\eta$ under different conditions. However, we also find interesting to consider a measure of the increase of $\eta$ by the existence of convective currents forcing the reactant to flow-through the membrane, which is defined as:

$$
\mathrm{E}=\frac{\eta\left(\phi^{2}, P\right)}{\eta\left(\phi^{2}, P=0\right)}
$$

Exact analytical solutions may be of very complex form for linear reactions (e.g. in a cylindrical membrane), and impossible to obtain for nonlinear ones. However, simplified analyses can be given in different regimes, namely:

Regime I: $\quad$ Kinetic control in nearly diffusive systems (section 3.1)

Regime II: Kinetic control under strong convective conditions (section 3.2)

Regime III: Diffusional control (section 4)

Regime IV: Convection dominated systems (section 5). 
The transitions between these regimes occur generically in an intermediate region and will be determined by the intersection of asymptotes for the effectiveness $(\eta)$ or enhancement ( E) factors.

\section{Chemical Regimes, $\phi^{2} \rightarrow 0$}

We first consider regimes where reaction is slow and transport is dominated by either diffusion (Regime I) or convection (Regime II). A perturbation procedure can be conceived for small values of the Thiele modulus, similarly to what was outlined in Lopes et al. [52, 53]. This analysis suggests writing the concentration profile inside the membrane, and hence the effectiveness factor, as a series of powers of $\phi^{2}$. The leading order term (of $\left.O(1)\right)$ in such series corresponds to the inert solution (no reaction) and is a function of the radial position, $c_{0}(r)$. The differential equations that allow the calculation of higher-order corrections to this term, make use of $c_{0}(r)$ in the subdominant kinetic term of the mass balance. The calculations can be considerably simplified if $c_{0}(r)$ is replaced by the averaged value over the cylindrical membrane thickness in the subproblem of $O\left(\phi^{2}\right)$, given by:

$$
\left\langle c_{0}\right\rangle=\frac{2 c_{2}(1+\varepsilon)^{2}+2\left(P_{C}-1\right)+\varepsilon\left(2+\varepsilon+P_{C}\right)}{(2+\varepsilon)\left(3 \varepsilon+P_{C}\right)}+\frac{1-c_{2}}{(1+\varepsilon)^{1+P_{C} / \varepsilon}-1} \frac{P_{C}+\varepsilon}{P_{C}+3 \varepsilon},
$$

for all values of $\varepsilon, c_{2}$ and $P_{C}$. In the case of a purely diffusive membrane:

$$
\left\langle c_{0}^{D}\right\rangle=\frac{c_{2}(1+\varepsilon)^{2}-1}{\varepsilon(2+\varepsilon)}+\frac{1-c_{2}}{2 \ln (1+\varepsilon)} .
$$

As we will see, the accuracy of this approximation increases as both surface concentrations become closer, i.e. $c_{2} \rightarrow 1$ (otherwise, the sweep phase conditions need to be strong, as negligible consumption of reactant occurs over the membrane). Diffusive conditions also lead to less accurate predictions, since convection tends to flatten out the profile, shifting the variation to a thin region downstream (hence, less contributing to the volume-averaged reaction rate). However, as we will see in the next section, these difficulties do not compromise seriously the accuracy of our approximate solutions. 
In the cases where the downstream boundary is closed to diffusion (Fig. 2B), $c_{0}(r)=\left\langle c_{0}\right\rangle$ (exactly) and is either equal to 1 or to $c_{2}$, whether the flow direction is outward or inward, respectively. The local conversion observed at the membrane exit is given by:

$$
\begin{aligned}
& X=\frac{(1+\varepsilon)^{1-P_{C} / \varepsilon}-(1+\varepsilon v)+v P_{C}}{\left(P_{C}-\varepsilon\right)\left(P_{C}+\varepsilon\right)} R(1) \phi^{2}+O\left(\phi^{4}\right) \\
& X=\frac{(1+\varepsilon)^{1+P_{C} / \varepsilon}-(1+\varepsilon v)-v P_{C}}{\left(P_{C}-\varepsilon\right)\left(P_{C}+\varepsilon\right)} \frac{R\left(c_{2}\right)}{c_{2}} \phi^{2}+O\left(\phi^{4}\right) \quad \text { (outward flow) }
\end{aligned}
$$

\subsection{Diffusive limit, $P \rightarrow 0$ (Regime I)}

Even when the convective contribution to the overall mass transfer can be considered small (due to low permeability or negligible pressure drop across membrane), few results have been reported for the reaction-diffusion problem with asymmetrical boundary conditions. Tan et al. [54] present the solution for a diffusive slab with first-order reaction in the context of partiallywetted catalyst particles in trickle-bed reactors. DeSimone et al. [55] considered the unsymmetrical nature of the solution for a diffusive membrane separating two reservoirs at different concentrations, while a reaction with order $m$ occurred in the slab. The effectiveness factor was written implicitly in terms of the concentration at a reference plane. Other works which also pertain to facilitated transport across planar films have been presented [56], but none addresses the problem as considered here, i.e. the approach and results are novel.

In this section, we derive approximate expressions for the effectiveness and enhancement factors which describe more comprehensive conditions than the ones found in previous works: (i) nonlinear kinetics; (ii) cylindrical porous wall with any value of the relative thickness, $\varepsilon$ (including thick membranes); (iii) concentration at both surfaces kept at any ratio, $c_{2}$; (iv) small convective effects, that will appear in a term of $O(P)$.

\subsubsection{Effectiveness factor for perfusive membranes (Fig. 2A)}

Using the asymptotic techniques described above, it is possible to show that: 
$\eta=\eta_{0}+\Lambda_{1} P-R\left(\left\langle c_{0}\right\rangle\right) R^{\prime}\left(\left\langle c_{0}\right\rangle\right) \Lambda_{2} \phi^{2}+O\left(\phi^{2}, \varepsilon^{2}, P^{2}\right)$.

Concerning the several terms in Eq.(8), it should be noted:

(a) The leading-order term $\eta_{0}$ concerns $\phi^{2} \rightarrow 0$ conditions in a membrane with prescribed values of $\varepsilon$ and $c_{2}$. This can be roughly approximated by $\eta_{0} \simeq R\left(\left\langle c_{0}^{D}\right\rangle\right)$, provided that $c_{2}$ is not too small. However, for power-law kinetics it is possible to obtain a better estimate by considering the exact solution of $c_{0}^{D}$. Details are shown in Appendix A and the final form can be written as

$$
\eta_{0}=\frac{(1+\varepsilon)^{\frac{2}{1-c_{2}}}}{2^{m+1} \varepsilon v}\left(\frac{1-c_{2}}{\ln (1+\varepsilon)}\right)^{m}\left[\Gamma\left(1+m, \frac{2 c_{2} \ln (1+\varepsilon)}{1-c_{2}}\right)-\Gamma\left(1+m, \frac{2 \ln (1+\varepsilon)}{1-c_{2}}\right)\right]
$$

for all values of $\varepsilon, m$ and $c_{2}$. In particular for a thin membrane ( $\varepsilon \rightarrow 0$ ), Eq.(9a) reduces to

$$
\eta_{0}=\frac{1}{1+m} \frac{1-c_{2}^{m+1}}{1-c_{2}}+\frac{\varepsilon c_{2}}{\left(1-c_{2}\right)^{2}} \frac{2-c_{2}^{m}\left(2+\left(1-c_{2}\right) m\right)-m\left(1-c_{2}\right) / c_{2}}{(m+1)(m+2)}+O\left(\varepsilon^{2}\right)
$$

The first term is the solution for a slab and, to the best of our knowledge, these results have never been reported before. The limit for a first order reaction $(m=1)$ is simply $\eta_{0}=\left(1+c_{2}\right) / 2$, in agreement with Tan et al. [54], followed by a small contribution for thin membranes of $O(\varepsilon)$ , equal to $\left(c_{2}-1\right) \varepsilon / 6$. In Fig. 3, we can see that $\eta_{0}$ is an increasing function of $c_{2}$ for several orders of reaction in thin $(\varepsilon=0.1)$ and thick $(\varepsilon=1)$ membranes. This is due to the diffusive loss of reactant (for $c_{2}<1$ ) through the surface at $r=1$, leading to values of effectiveness below 1 . In agreement with what is known for catalytic particles with $\phi^{2}>0$, the effectiveness when $\phi^{2} \rightarrow 0$ also decreases as the order of reaction or membrane thickness increase. Hence, the value of $\eta_{0}$ depends on the membrane geometry and on the concentrations that prevail at both surfaces, but naturally not on the actual reaction rate. However, it is curious that in the limit of a very slow reaction, the effectiveness depends on the form of the reaction rate law (namely, on kinetic parameters such as the order of reaction $m$ ), since the first estimate to the concentration profile (given by $c_{0}$ ) is kinetic-independent. The aforementioned dependency is not a consequence of reaction nonlinearity, but of the asymmetry in the boundary conditions of the transport-reaction problem. This happens since the first estimate to the effectiveness factor ( 
$\eta_{0}$ ) derives from the $O\left(\phi^{2}\right)$ correction in the perturbation series, which is calculated writing the reactive term in the mass balance as $R\left(c_{0}\right)$, which is different from 1 . In the case of equal conditions at both surfaces ( $c_{2}=1$ ), the effectiveness factor approaches $\eta \rightarrow 1$, for any reaction rate.

In a diffusive hollow fibber reactive membrane with the so-called 'normal kinetics' (yielding monotone variation of the effectiveness factor with the Thiele modulus), $\eta_{0}$ is the maximum effectiveness factor that can be attained. In Appendix A, we also discuss the influence of these results in the formulation of criteria to exclude internal mass transfer resistance (a fundamental step when using experimental data to evaluate the reaction intrinsic kinetics in a membrane reactor setup).

(b) Eq.(9) does not include information on the convective process. The main correction accounting for these effects can be given by the $O(P)$ term in the expansion of $R\left(\left\langle c_{0}\right\rangle\right)$ for small $P$. This is written as the second parcel in Eq.(8), where:

$$
\Lambda_{1}=\left.\left(R^{\prime}\left(\left\langle c_{0}\right\rangle\right) \frac{d\left\langle c_{0}\right\rangle}{d P}\right)\right|_{P=0}
$$

Note that the kinetic-independent expression for $\left\langle c_{0}\right\rangle$ is given in Eq.(6a) and that $R^{\prime}\left(\left\langle c_{0}\right\rangle\right)=d R /\left.d c\right|_{\left\langle c_{0}\right\rangle}$. For generic order of reaction $m$ and any value of $\varepsilon$,

$$
\Lambda_{1}=\frac{\left(1-c_{2}\right) m}{2^{m+1} \varepsilon^{2} \ln (1+\varepsilon)}\left[2 \frac{c_{2}(1+\varepsilon)^{2}-1}{\varepsilon(2+\varepsilon)}+\frac{1-c_{2}}{\ln (1+\varepsilon)}\right]^{m-1}\left[\left(\frac{1}{v}+\varepsilon\right) \ln (1+\varepsilon)-\varepsilon\right]
$$

For small $\varepsilon, \Lambda_{1}$ becomes:

$$
\Lambda_{1}=\frac{m}{9} \frac{1-c_{2}}{2^{m+2}\left(1+c_{2}\right)^{2-m}}\left[6\left(1+c_{2}\right)-\varepsilon\left(1+5 c_{2}\right)-2\left(1-c_{2}\right) m \varepsilon\right]
$$

This contribution is positive for $c_{2}<1$ and $P>0$ as expected. It is zero, whenever the concentrations at both surfaces are equal, since the leading-order result for the effectiveness factor is 1 , regardless of the degree of convective transport. 
(c) The dependence on the Thiele modulus is captured by considering a higher-order term (of $O\left(\phi^{4}\right)$ ) in the perturbation procedure, which uses the averaged values of the initial guess for the concentration profile, $\left\langle c_{0}\right\rangle$. This results in the last term of Eq.(8), where the geometric factor is:

$$
\Lambda_{2}=\frac{1}{8 \varepsilon^{2}}\left[2+(2+\varepsilon) \varepsilon-\frac{(2+\varepsilon) \varepsilon}{\ln (1+\varepsilon)}\right] \simeq \frac{1}{12}\left[1+O\left(\frac{\varepsilon^{2}}{60}\right)\right] .
$$

As expected, for 'normal kinetics' the effectiveness decreases with $\phi^{2}$. This asymptotic approximation is compared with numerical results in Fig. 4. The same approach can be extended for systems with reactant inhibition, provided that the correct value of $\eta_{0}$ is known. Then, the behavior for $\phi^{2}<1$ is well predicted by the higher-order corrections in Eq.(8).

\subsubsection{Enhancement factor for perfusive membranes (Fig. 2A)}

Using the same reasoning, it is possible to calculate $\mathrm{E}$ as

$$
\mathrm{E} \simeq \frac{R\left(\left\langle c_{0}\right\rangle\right)}{R\left(\left\langle c_{0}^{D}\right\rangle\right)} \simeq 1+\left(1-c_{2}\right) \frac{\Lambda_{0}}{2} m P+O\left(P^{2}\right),
$$

where, $\Lambda_{0}=\frac{(1+\varepsilon v) \ln (1+\varepsilon)-\varepsilon v}{\left(1-c_{2}\right) \varepsilon^{2} v-\left[1-c_{2}(1+\varepsilon)^{2}\right] \varepsilon \ln (1+\varepsilon)}$.

The last term in (12a) is written for power-law kinetics of order $m$ and small $P$. When $\varepsilon \rightarrow 0$, the geometric factor simplifies to:

$$
\mathrm{E} \simeq 1+\frac{1-c_{2}}{1+c_{2}} \frac{m P}{6}\left[1-\frac{1+5 c_{2}}{1+c_{2}} \frac{\varepsilon}{6}+O\left(\varepsilon^{2}\right)\right]+O\left(P^{2}\right) .
$$

A higher-order term (of $O\left(\phi^{2} P\right)$ ) could be considered in (12a) to obtain the dependence on the surface reaction rate, but this is generally not required as the variation of $\mathrm{E}$ with $\phi^{2}$ is negligible for small values of this parameter (say, $\phi^{2}<1$ ). We only find this additional term useful to distinguish between the cases where the curve $\mathrm{E}\left(\phi^{2}\right)$ is increasing or decreasing. It is possible to observe that $\mathrm{E}\left(\phi^{2}\right)$ is a decreasing function for $m>1$ (i.e. $\mathrm{E}_{\max }=\mathrm{E}\left(\phi^{2} \rightarrow 0\right)$ ), but an increasing one for $m<1$. These conclusions are valid for small values of $P$. 


\subsubsection{Solutions for catalytic filtration (downstream closed to diffusion, Fig. 2B)}

The previous results can be obtained more easily in the case where the boundary condition on the side where the convective flow exits the membrane is replaced by a no-flux requirement (Fig. 2B). The results for the effectiveness and enhancement factors are presented in Table 1.

\subsection{Convective limit, $P \rightarrow \infty$ (Regime II)}

Under appreciable convective conditions, the effectiveness and enhancement factors are given by:

$$
\eta=R\left(\left\langle c_{0}\right\rangle\right)\left[1-\frac{\Lambda_{\infty}}{2} R^{\prime}\left(\left\langle c_{0}\right\rangle\right) \frac{v \phi^{2}}{\left|P_{C}\right|}\right]+O\left(\frac{\phi^{4}}{P^{2}}\right) .
$$

Here, $\left\langle c_{0}\right\rangle=1$ or $c_{2}$, whether the flow is outward or inward, respectively. This expression also holds when the downstream surface is closed to diffusion. Note that Thiele and Peclet numbers appear grouped in the subdominant correction, a feature of convective-dominated solutions that we will reencounter in section 5. Rigorously, the constant in Eq.(13) is

$$
\Lambda_{\infty}=\frac{(1+\varepsilon)^{2\left(1+P_{C} / \varepsilon\right)}-1}{\left[(1+\varepsilon)^{1+P_{C} / \varepsilon}-1\right]^{2}}
$$

but $\Lambda_{\infty} \rightarrow \pm 1$ fast when $P_{C} \rightarrow \pm \infty$. This result is valid for any condition specified downstream, due to the importance of convective transport in this regime. An estimate of the maximum enhancement factor observed in this regime is calculated according to:

$$
\mathrm{E}=\frac{R\left(\left\langle c_{0}\right\rangle\right)}{\eta_{0}}
$$

which simplifies to

$\mathrm{E} \simeq \frac{1}{\eta_{0}}$ (in outward flow) or $\mathrm{E} \simeq \frac{R\left(c_{2}\right)}{\eta_{0}}$ (in inward flow)

A correlation merging the limits represented by Eqs.(12) and (14) can be proposed as:

$$
\mathrm{E}=1-\left(1-\frac{1}{\eta_{0}}\right)\left(\frac{P}{1+P}\right)^{n} \quad \text { (outward flow; small } \varepsilon \text { ). }
$$


The value of the exponent $n$ was chosen in order to minimize the sum of the square of the deviations between Eq.(15) and numerical results for the worst case scenario $\left(c_{2}=0\right)$. A value around $n=3.3$ was found to yield a good agreement in the limit of small $\varepsilon$. This correlation is plotted in Fig. 5a for $m=2$, where the maximum relative error of this prediction is around $4 \%$. It is particularly useful to describe an intermediate range of Peclet numbers. This is also shown in Fig. 5 c for kinetics with $m<1$ and low Thiele modulus $\left(\phi^{2}=0.1\right)$.

In Eq.(14), only the leading-order terms for $\eta$ were considered. Higher-order terms do not improve the approximation, but are useful to make conclusions regarding the monotonicity of the $\mathrm{E}\left(\phi^{2}\right)$ function. Thus, a maximum in enhancement exists as the reaction rate is increased, if the Peclet number is allowed to increase above the following criterion:

$$
P_{C}>\frac{3}{4 \Lambda_{2}}\left\langle c_{0}^{D}\right\rangle^{1-m} \quad \text { (outward flow) }
$$

According to Eq.(16) for $\varepsilon=0.1$ and $m=2,9 \leq P_{C} \leq 18$ for $1 \geq c_{2} \geq 0$. For the case of inward flow, this indicative value can be derived similarly from previous results. Comparing this prediction with numerical calculations, an agreement in the order of magnitude of $P_{C}$ is observed, as well as in the trends resulting from parameter variation in Eq.(16). For orders of reaction greater than 1 , the value of $P_{C}$ decreases as $c_{2}$ increases. For $m>1$, advantage from catalyst effectiveness at higher reaction rates is only achieved if $P_{C}$ exceeds this value, which for a second-order reaction is around 10, as can be seen in Fig. 5 (curve $\mathrm{E}_{\max } @ \phi^{2} \neq 0$ ). The

restriction that this requirement poses on $P_{C}$ is not too serious if $m<1$ (since $\left\langle c_{0}^{D}\right\rangle<1$ ), and it is likely that the behavior of $\mathrm{E}\left(\phi^{2}\right)$ near $\phi^{2} \rightarrow 0$ is that of an increasing function (the same was understood from the behavior at low Peclet numbers).

\subsection{Onset of significant convective effects in kinetic control (Regimes I $\rightarrow$ II)}

From the point of view of the membrane reactor, the assessment of the importance of convective effects should not be based on the magnitude of $P$ alone (or permeability values), since the observed enhancement is also a function of the reaction rate. Under kinetic control 
(small $\phi$ ), the intersection of the asymptotes in the $\mathrm{E}-\phi^{2}$ plot for regimes I and II yields the following transition value for the Peclet number $\left(P^{*}\right)$ :

$P^{*}=\frac{2}{\left(1-c_{2}\right) m} \frac{1-\eta_{0}}{\Lambda_{0} \eta_{0}}$ where $\mathrm{E}\left(P^{*}\right)=\frac{1}{\eta_{0}}$

The $\mathrm{E}\left(P^{*}\right)$ curve is represented in Fig. 5 (a) and (b) as a dashed line separating the two regimes. The limit of small $\varepsilon$ can be employed to further simplify (17a) to:

$$
P^{*}=\frac{6}{m} \frac{1-\eta_{0}}{\eta_{0}}\left(\frac{1+c_{2}}{1-c_{2}}\right) .
$$

Some limiting situations are not described by this transition $\left(c_{2}=1, m=0, \eta_{0}=1\right)$ since the higher-order terms from which (17) was derived, cease to exist in those cases. However, it is possible to understand that as the order of reaction decreases, the diffusive regime I becomes more appropriate to describe the enhancement factor. This can be inferred from Fig. 5c.

\section{Diffusional REgIME, $\phi^{2} \rightarrow \infty$ (REgIME III)}

\subsection{Effectiveness and enhancement}

When reaction kinetics is faster than mass transport, a concentration boundary layer may occur near one or both surfaces of the membrane. These regions are described by a reactiondiffusion leading-order balance [57], followed by a correction accounting for curvature and convection [53]. The effectiveness factor calculated from Eq.(4) for arbitrary kinetics and specified surface concentrations is given by

$$
\eta=\frac{K_{\infty}}{\phi v}+\tilde{K}_{\infty} \frac{P_{C}}{v \phi^{2}}+\left(1-c_{2}\right) \frac{P}{v \phi^{2}}+O\left(\phi^{-3}\right)
$$

where the following factors were defined (the last term is written for power-law kinetics):

$$
\begin{aligned}
& K_{\infty}=(1+\varepsilon) \sqrt{2 \int_{0}^{c_{2}} R\left(c^{\prime}\right) d c^{\prime}}+\sqrt{2 \int_{0}^{1} R\left(c^{\prime}\right) d c^{\prime}}=(1+\varepsilon) \sqrt{\frac{2 c_{2}^{m+1}}{m+1}}+\sqrt{\frac{2}{m+1}} \text { and } \\
& \tilde{K}_{\infty}=\int_{0}^{c_{2}} \frac{R\left(c^{\prime}\right)}{R\left(c_{2}\right)} d c^{\prime}-\int_{0}^{1} R\left(c^{\prime}\right) d c^{\prime}=\frac{c_{2}-1}{m+1} .
\end{aligned}
$$


Eq.(18) includes contributions from the solutions near $r=0$ and $r=1$. If the exit of the membrane reactor is closed to diffusion, then $c_{2}=0$ for outward flow. On the other hand, for inward flow: $K_{\infty}=(1+\varepsilon) \sqrt{2 \int_{0}^{c_{2}} R\left(c^{\prime}\right) d c^{\prime}}$ and the term $P /\left(\phi^{2} v\right)$ should be subtracted from Eq.(18). If the concentration at one of the surfaces is kept at negligible values (e.g. $c_{2} \rightarrow 0$ ), then there is also no boundary layer on that side of the membrane (i.e. the dead core extends from $\hat{r} \sim a+O\left(t_{m} / \phi\right)$ up to the outer surface at $\left.\hat{r}=a+t_{m}\right)$. The effect of convection is to slightly increase the fraction of reactant near the surface through which the flow enters the membrane, increasing the reactant penetration depth by $O(P / \phi)$. The purely diffusive limit of Eq.(18) is given by

$\eta_{\infty}=\frac{K_{\infty}}{\phi v}-\frac{\tilde{K}_{\infty} \varepsilon}{v \phi^{2}}+O\left(\phi^{-3}\right)$

These results allow the calculation of the asymptote of $\mathrm{E}$ (performance enhancement due to convection) as $\phi /|P| \rightarrow \infty$ :

$$
\mathrm{E}=1+\frac{1-c_{2}+\tilde{K}_{\infty}}{K_{\infty}} \frac{P}{\phi}+O\left(\phi^{-2}\right)
$$

The enhancement factor $\mathrm{E}$ is a decreasing function of $\phi$, approaching 1 as diffusion becomes more limiting. Note that the order of the correction in the enhancement factor is $O(P / \phi)$, which can also be written as a Peclet number where the length scale is the thickness of the concentration boundary layer (given in this regime by $\sim t_{m} / \phi$ ) instead of the total membrane thickness $t_{m}$.

For estimation purposes, the effectiveness of a diffusive membrane can be approximated by an expression taking into consideration the asymptotes given in Eqs.(9) and (20). This approximation should be able to reasonably describe $\eta$ for all values of Thiele modulus. It is given by:

$$
\eta^{n}=\eta_{\infty}^{n} \tanh \left(\frac{\eta_{0}^{n}}{\eta_{\infty}^{n}}\right)
$$


Eq.(22) and the asymptotes from which it was constructed are plotted in Fig. 6 and compared with the numerical results obtained with gPROMS $^{\circledR}$. The exponent $n$ was set to 0.8 in order to describe the lower values of $c_{2}$, but the approximation describes reasonably the whole range of the effectiveness factor (including intermediate values) for other values of $n$ close to 0.8 .

The correlation given in Eq.(22) can be useful even for linear kinetics, since the generic solution for a cylindrical thick membrane with unequal surface concentrations is complex to obtain by analytical means. For the most unfavorable case $\left(\varepsilon=1\right.$ and $\left.c_{2}=0\right)$, using $n=0.8$ yields a maximum relative error around $2 \%$ (had we used $n=1$, the maximum error would be $5 \%)$.

\subsection{Reaction-diffusion intermediate region (Regimes I $\rightarrow$ III)}

We find the intersection defined by Eqs.(12) and (21) to determine the transition between chemical and diffusional asymptotes, in the presence of weak to moderate convection. Taking these limits, the value of the transition Thiele modulus $\phi^{*}$ is actually independent of $P$, and is calculated from:

$\phi^{*}=\frac{2}{m \Lambda_{0}} \frac{1-c_{2}+\tilde{K}_{\infty}}{\left(1-c_{2}\right) K_{\infty}}$,

which is plotted in Fig. 7. Eq.(23a) is not applicable in the cases where higher-order estimates to the enhancement factor cancel out (which happens for zero-order kinetics, symmetric boundary conditions, etc.). In these cases, the intersection of effectiveness factors may be of interest. In the diffusional limit, it writes as

$\eta=\frac{K_{\infty}}{\phi v}=\eta_{0}$, yielding: $\phi^{*}=\frac{K_{\infty}}{\eta_{0} v}$

This is plotted in Fig. 4, where $\left(\phi^{2}\right)^{*}=6$. We note that when asymmetry in the boundary conditions exists, the expected enhancement of the effectiveness factor in the intermediate range of Thiele modulus may not be observed, contrary to what was found for uniform surface concentration around a catalytic pellet with 'large pores' (e.g. as predicted in the analysis of 
[1]). Actually, as seen in Fig. 7, the improvement may decrease continuously with $\phi^{2}$. In these cases, maximum advantage is taken from the regimes under kinetic control.

\subsection{Intermediate convection-diffusion-reaction region (Regimes II $\rightarrow$ III)}

Regimes II and III can be considered to be opposite to each other in the $\left(\phi^{2}, P\right)$ space. While in the former, convection dominates at leading-order across the full membrane length, in the latter these effects are restricted to small contributions (of $O(P / \phi)$ ) in boundary layers near the surface. On the other hand, as the Thiele modulus is increased (regime changes from II to III), the dominance of the reaction term increases from being negligible, to being unmatched by any other mechanism in the 'dead core' region that is established in most of the membrane. The transition between the two pictures occurs in the intermediate region where convection, diffusion and reaction all play a role. Intersecting the asymptotic expressions for E (making use of correlation (15) for Regime II in the intermediate range of $P$ ), the following transition is obtained:

$\phi^{*}=\frac{(1+P)^{n}}{P^{n-1}}\left(\frac{1-c_{2}+\tilde{K}_{\infty}}{K_{\infty}} \frac{\eta_{0}}{1-\eta_{0}}\right)$.

The numerical coefficient in the right hand side (inside brackets) is $O(1)$, and a reasonable value for $n$ is around 3 (see section 3.2). The limit of Eq.(24) at moderate to high $P$ is the behavior of interest for $\phi^{*}(P) \sim P$. This scaling rule will be discussed in section 6 , when the complete regime mapping is presented.

\section{CONVECTIVE REgIME, $P \rightarrow \infty$ (REgIME IV)}

The structure of the concentration profile in a strong convective regime consists on the same two domains found in Lopes et al. [52]. Briefly, convection is balanced by reaction in most of the membrane (starting at the inlet surface), with the latter conceding its place to diffusion near the membrane exit. For arbitrary kinetics, it is important to remark the following features of the perturbation solutions: 
(i) the solution in the global (outer) scale reaction-dominated region, which extends from the inlet surface up to a radial position $\hat{r} \sim O\left(t_{m}\right)$, has different forms depending on the reaction rate law, and

(ii) at leading order, the general solution at the diffusive boundary layer near the downstream surface (with thickness $\hat{\delta} \sim D_{\text {eff }} /\langle u\rangle$ ) does not include information on the reaction.

Naturally, in a high Peclet number regime, $\hat{\delta}<<t_{m}$ and convection is important in the whole spatial domain. According to Prandtl's principle [58], the generic composite profile between the two surfaces kept at specified concentrations is:

$$
\begin{aligned}
& c(r)=c_{\text {out }}(r)+\left(c_{2}-\left.c_{\text {out }}\right|_{r=1}\right) \exp \left[\frac{-P_{C}}{1+\varepsilon}(1-r)\right]+O\left(P_{C}^{-1}\right)\left(\text { outward flow, } P_{C} \rightarrow \infty\right) \\
& \left.c(r)=c_{\text {out }}(r)+\left(1-\left.c_{\text {out }}\right|_{r=0}\right) \exp \left[P_{C} r\right]+O\left(P_{C}^{-1}\right) \quad \text { (inward flow, } P_{C} \rightarrow-\infty\right) .
\end{aligned}
$$

In Eqs.(25), the value of the concentration profile at the global scale $\left(c_{\text {out }}\right)$ is required. This will be determined in the following section.

\subsection{Concentration distribution at the global scale, $c_{\text {out }}(r)$}

The solution for this region of the membrane is given implicitly in terms of the incomplete beta function $B_{z}(a, b)$ for kinetic expressions as in Eq.(2), or using the Lambert $W$ function for the special case of Michaelis-Menten kinetics. However, for our purposes it is more convenient to consider different degrees of reactant inhibition in the reaction rate law. Moreover, as we shall see, the relevant quantity for the calculation of the effectiveness and enhancement factors is $c_{\text {out }}$ evaluated at the downstream surface (given in Table 2). Apart from the kinetic parameters, $c_{\text {out }}$ is also a function of $\varphi$, a "flow-through membrane Damköhler number", which is given by:

$$
\varphi=\frac{v \phi^{2}}{P_{C}}\left(1+k_{i}\right)^{p} .
$$


In some cases, expressions for the concentration distribution in most of the membrane (except in the boundary layer) can be obtained in a straightforward manner (e.g. in the absence of inhibition, for orders of reaction $m \neq 1$ ).

\subsection{Effectiveness and Enhancement factors for perfusive membrane (Fig. 2A)}

The effectiveness factor can be calculated using Eqs.(4) and (25). However, the contribution from the boundary layer to the flux at the inlet surface is a transcendentally small term of $O\left(e^{-\left|P_{c}\right|}\right)$, and can be ignored. Thus, for outward flow $(P \rightarrow \infty)$ :

$$
\eta=\frac{1-\left.c_{\text {out }}\right|_{r=1}}{\left(1+k_{i}\right)^{-p} \varphi}+\frac{\left(1-c_{2}\right) \varepsilon}{v \phi^{2}}+\frac{1}{v P_{C}}\left[R(1)-(1+\varepsilon)^{2} R\left(\left.c_{\text {out }}\right|_{r=1}\right)\right]+O\left(e^{-P_{C}}\right)
$$

while for inward flow $(P \rightarrow-\infty)$ :

$$
\eta=\frac{c_{2}-\left.c_{\text {out }}\right|_{r=0}}{-\left(1+k_{i}\right)^{-p} \varphi}+\frac{\left(1-c_{2}\right) \varepsilon}{v \phi^{2}}+\frac{1}{v P_{C}}\left[R\left(\left.c_{\text {out }}\right|_{r=0}\right)-(1+\varepsilon)^{2} R\left(c_{2}\right)\right]+O\left(e^{P_{C}}\right) .
$$

The last term in Eq.(33) is $O\left(P_{C}^{-1}\right)$ and therefore subdominant in this regime. These results may indicate, for example, how effective the consumption of oxygen by cells growing in a radial-flow perfusive bioreactor is for Michaelis-Menten kinetic law. Fig. 8 shows the variation of the effectiveness factor with the dimensionless reaction rate in the regimes found under strong convective conditions. Different levels of substrate inhibition (from weak to moderate) are considered. Highly inhibited kinetics presents a more complex behavior, including the appearance of multiple steady states and values of effectiveness above 1 (hence $\eta\left(\phi^{2}\right)$ is a nonmonotonic function). However, the chemical and diffusional limits are still approached, since the 'abnormal' effects are usually in the intermediate range of $\phi^{2}$. Therefore, it is expected that the results in this section (including the ones below, defining maximum enhancement) also apply to this situation.

The enhancement factor follows from Eq.(5), using the previously derived solutions: $\eta(P)$ from Eqs.(33) and $\eta(P=0)$ from Eqs.(22) or (18) for strongly inhibited systems. When $\varphi \sim 1$ (balance between the timescales for reaction and convection), the Thiele modulus will also be large and 
$\mathrm{E} \simeq \frac{c_{\text {in }}-\left.c_{\text {out }}\right|_{\text {exit }}}{K_{\infty}\left(1+k_{i}\right)^{-p}} \frac{v \phi}{|\varphi|}$

which means that, at leading-order, $\mathrm{E} \sim \frac{O(\varphi)}{|\varphi|} \phi \sim \sqrt{\varphi P_{C}}>>1$. The result for $\mathrm{E}$ derived from Eqs.(33) and (22) (with $n=1$ ) is represented along with the predictions for the other high Peclet number regimes in Figs. 9 and 10.

\subsection{Maximum enhancement}

As we have seen in section 3.2, the analysis of Regime II $\left(\phi^{2} / P_{C}<<1\right)$ suggests that when the Peclet number exceeds the value given by Eq.(16), maximum enhancement is observed at nonzero Thiele modulus. Since $\mathrm{E}$ behaves as a decreasing function of $\phi^{2}$ in Regime III $\left(\phi / P_{C}>>1\right)$, a maximum in the $\mathrm{E}\left(\phi^{2}\right)$ curve appears in a region of finite $\phi^{2} / P_{C}$. At sufficiently high values of $P_{C}$, this region is characterized by the scaling rule for Regime IV: $\phi^{2} / P_{C} \sim O(1)$. This scaling relationship indicates that under these conditions, the Thiele modulus will not be much higher than the Peclet number, i.e. they are at most comparable. The nonlinearity of the reaction rate law suggests a different treatment than the one strictly required for first-order reactions. Furthermore, we note that: $(i)$ as seen in Eqs.(27), the integral of concentration when solving the convective-dominated mass balance writes differently whether $m=1$ or $m \neq 1$; (ii) due to the approximate nature of the analysis, results derived originally for linear kinetics may not be reproduced exactly by taking the $m \rightarrow 1$ limit for arbitrary kinetics; (iii) solutions for nonlinear kinetics will require the use of a different asymptotic expansion. Nevertheless, in the cases where it is possible to establish a direct comparison, good agreement is observed, as shown below. 


\subsubsection{Power-law kinetics}

It is possible to understand that the maximum of $\mathrm{E}$ with respect to $\phi$ is calculated from a highly nonlinear algebraic equation (obtained by setting the derivative of the enhancement factor with respect to $\phi^{2}$ to zero) in the dimensionless group $\varphi=v \phi^{2} / P_{C}$ :

$$
\eta+2 \varphi \frac{d \eta}{d \varphi}=0
$$

At leading-order (see Eq.(33)), this expression is given by

$$
\left.c_{\text {out }}\right|_{\text {exit }}-2 \varphi \frac{\left.d c_{\text {out }}\right|_{\text {exit }}}{d \varphi}=c_{\text {in }},
$$

where $c_{i n}$ is the inlet concentration ( $=1$ or $\left.c_{2}\right)$ and $\left.c_{\text {out }}\right|_{\text {exit }}$ is the value of the outer profile at the exit surface (given in Table 2), both normalized by the concentration at $r=0$. An additional term of $O\left(P_{C}^{-1}\right)$ should be added to Eq.(36), which in general yields more complex expressions, without improving the estimates significantly. It will be considered only for simpler cases (power law kinetics) as described below.

In order to explicitly understand the effect of the kinetic parameters in the optimum value of $\varphi$, instead of solving this expression numerically, we seek an approximate solution using the scaling rule as initial guess. When enhancement is maximum, it is reasonable to expect that the relationship $\varphi \sim 1$ will remain valid, regardless of the form of the reaction rate law (without significant inhibition). Thus, we assume that the solution can be written as:

$\varphi \simeq 1+\varphi_{0}+\frac{\varphi_{1}}{P_{C}}$

where $\varphi_{0}$ and $\varphi_{1}$ depend on $m, p$ and $k_{i}$. Also, $\varphi_{0}<<1$ and $P_{C} \rightarrow \infty$ (i.e. the two last terms are subdominant). Introducing Eq.(37) into the nonlinear equation (36) and expanding for small $\varphi_{0}$ and $P_{C}^{-1}$, it is possible to calculate both coefficients in the expression for $\varphi$. Table 3 includes these results for some kinetics. The leading-order estimate $\varphi_{0}$ does not include information on the downstream concentration, due to the assumed convective dominance. For power-law kinetics, these predictions are used to indicate the optimum enhancement points in Figs. 9 and 10. Note that the limit of Eq.(46) for a first-order reaction is given by: 


$$
\varphi \simeq 1.220+\frac{2.121}{v P_{C}}\left[1-\frac{(1+\varepsilon)^{2}}{2.7183}-\varepsilon v\left(1-c_{2}\right)\right] .
$$

Comparing this limit with the result derived specifically for a first-order reaction (given in Eq.(45 )), the deviation in the leading-order term is only of 3\%, while the subdominant term of $O\left(P_{C}^{-1}\right)$ is underestimated. The respective value of the enhancement for $m=1$ should, however, be calculated as detailed elsewhere [49].

\subsubsection{Maximum enhancement line}

It is also possible to estimate the maximum enhancement at high Peclet number $\left(\mathrm{E}_{\max }\right)$, that is observed for the optimum values of $\varphi_{\text {opt }}$ derived above. The leading-order term of $\mathrm{E}$, given by Eq.(34), describes reasonably well the loci of maxima in the enhancement factor curves. This is plotted in Fig. 9 as the $\mathrm{E}_{\max }\left(\phi^{2}\right)$ line. The value of $\mathrm{E}$ which is the coordinate of the optimum enhancement points is more accurately calculated using the estimate for regime IV, given by $\mathrm{E}_{\text {opt }}=\eta\left(\varphi_{\text {opt }}\right) / \eta_{D}$, where $\eta\left(\varphi_{\text {opt }}\right)$ is the effectiveness factor evaluated at $\varphi_{\text {opt }}$ (according to Eq.(33)) and $\eta_{D}$ is the diffusive solution (for $P=0$, given e.g. by the correlation presented in Eq.(22) with $n=1$ ). For high $P_{C}$, it is enough to consider the leading-order term in Eq.(33), as shown in Fig. 10. A very good agreement with numerical results is obtained for values of $c_{2}$ and $\varepsilon$ covering the whole range of interest.

\subsubsection{Maximum enhancement window}

The region around the maximum in the enhancement curves extends from the intersection of $\mathrm{E}_{\max }\left(\phi^{2}\right)$ with the chemical convective-dominated regime (Regime II), up to the one with the diffusion controlled regime (Regime III), i.e. $\phi_{*}<\phi<\phi^{*}$. For power-law kinetics (outward flow), significant consumption of reactant in a convection-dominated regime occurs for $\phi>\phi_{*}$, where 
$\phi_{*}=\frac{R\left(\left\langle c_{0}\right\rangle\right)}{v \eta_{0}} \frac{K_{\infty} \varphi_{o p t}}{1-\left[1+(m-1) \varphi_{o p t}\right]^{1 /(1-m)}}$.

On the other hand, the convection changes from being important at the global scale to being restricted to boundary layers when $\phi>\phi^{*}$ :

$$
\phi^{*}=\frac{\varphi_{o p t}}{2 v} \frac{\tilde{K}_{\infty} P_{C}+2\left(1-c_{2}\right) P}{1-\left[1+(m-1) \varphi_{o p t}\right]^{1 /(1-m)}} .
$$

The window of values of the Thiele modulus is identified in Fig. 9 for a second order reaction.

\subsubsection{Zero-order reaction}

The special case of a zero-order reaction is of relevance as the high substrate concentration asymptote in Michaelis-Menten rate law. The effect of intraparticular flow in systems where the rate of reactant consumption is nearly independent of its concentration was considered by several authors. Rodrigues et al. [59] studied analytically the isothermal problem in a slab with both surfaces exposed to the same concentration, while Stephanopoulos et al. [60] presented numerical results for spherical geometry. Lopes et al. [52] also considered the planar shape under non-isothermal conditions. Moreover, they plotted the analytical solutions for the isothermal problem [59] in a $P-\phi^{2}$ map, showing several iso-effectiveness factor lines, as well as the curve which delimits the situations where concentration annulment inside the slab occurs or not (the iso-effectiveness factor curve for $\eta=1$ ). Here, we just consider some aspects of the analysis for asymmetrical boundary conditions, which will help to understand the nature of the optimum solutions found for other reaction rate laws.

We start by considering the case where no reactant starvation occurs, to show that the position in the slab where concentration reaches its minimum value is given by:

$$
r_{\min }=\frac{1}{P} \ln \left[\frac{e^{P}-1}{P} \frac{\phi^{2}}{\phi^{2}-\left(1-c_{2}\right) P}\right] .
$$


Eq.(40) shows that a dependence of $r_{\min }$ on $\phi^{2}$ is introduced if $c_{2} \neq 1$, otherwise the result from [59] is recovered. The critical value of the Thiele modulus (above which $c\left(r_{\min }\right)=0$ ) can be calculated in the diffusive and convective limits as:

$$
\begin{array}{ll}
\phi_{C}^{2}=2\left(1+\sqrt{c_{2}}\right)^{2}, & \text { when } P \rightarrow 0 \\
\phi_{C}^{2}=P \frac{P-\left(1-c_{2}\right)}{\ln \left(e^{P}-1\right)-\ln (P)-1} \simeq P+\ln (P)+1, & \text { when } P \rightarrow \infty .
\end{array}
$$

Note that Eq.(41b) can be written as $\varphi_{C}=\phi_{C}^{2} / P \simeq 1+1 / P$ when $P \rightarrow \infty$, which is the same structure predicted for the value of $\varphi$ which maximizes E for any kinetic expression (see Eq.(37)). Therefore, we may say that the conditions for maximum enhancement of the catalyst effectiveness correspond to those where (near) concentration annulment occurs for the first time inside the membrane (as $\phi^{2}$ is increased). For $\phi^{2}<\phi_{C}^{2}$, no exhaustion occurs and the effectiveness is always equal to 1 (independent of $c_{2}$ as expected for zero-order kinetics). When concentration annulment inside the membrane occurs, the following results for effectiveness (with and without convective effects) are obtained, which do not seem to have been reported before $\left(c_{2}<1\right)$ :

$$
\begin{array}{ll}
\eta=\frac{P}{\phi^{2}}+\frac{1}{P}\left(1+\frac{\phi^{2}}{c_{2} P^{2}}\right) \ln \left(1+\frac{c_{2} P^{2}}{\phi^{2}}\right)+\frac{1}{P} \frac{2-e^{1+P^{2} / \phi^{2}}}{1-e^{1+P^{2} / \phi^{2}}} \quad \text { (strong convective flow) } \\
\eta=\frac{\sqrt{2}}{\phi}\left(1+\sqrt{c_{2}}\right) & \text { (diffusive conditions). }
\end{array}
$$

The enhancement factor as a function of $\phi^{2}$ presents three distinct regions: $(a)$ for values below that in (41a), $\mathrm{E}=1 ;(b)$ for $\phi^{2}$ between the values in Eqs.(41a) and (41b), E is the reciprocal of Eq.(42b), hence an increasing function of $\phi$; and (c) for $\phi>\phi_{C}$ (given by Eq.(41 b)), the ratio between Eqs.(42), yields a decreasing function of $\phi$. The maximum enhancement should occur at the maximum value of $\phi^{2}$ which is still under chemical control through the action of convection (given in Eq.(41b)), while the corresponding diffusive system is severely mass transfer limited (Thiele modulus above that given in Eq.(41a)): 
$\mathrm{E}_{\max }=\frac{\phi_{C}}{\sqrt{2}\left(1+\sqrt{c_{2}}\right)}=\frac{1}{1+\sqrt{c_{2}}} \sqrt{\frac{P+\ln (P)+1}{2}}$,

which has the same dependence found in Eq.(34): $\mathrm{E}_{\max } \sim \phi \sim \sqrt{P}$.

\subsubsection{Cases with reactant inhibition}

The approximate analytical procedure outlined above is also able to handle the more complex situation where reactant inhibition is present. Considering kinetics with MichaelisMenten type, it is possible to show that in the limit of weak inhibition $\left(k_{i} \rightarrow 0\right)$ : $\varphi_{0} \sim 3-e+1.5 k_{i}+O\left(k_{i}^{2}\right)$. This was obtained for outward flow using Eqs.(29) and (36) with $\varphi \simeq 1+\varphi_{0}$, since at high Peclet number $\varphi_{1}$ can be ignored (it would only lead to cumbersome algebraic manipulations). For inward flow, the result in the same limit is given by $\varphi_{0} \sim 3-e+1.5 k_{i} c_{2}+O\left(k_{i}^{2}\right)$, and it should be introduced in an expression for $\varphi$ (which now takes negative values owing to the fact that $\left.P_{C}<0\right)$, such as: $\varphi=-1-\varphi_{0}$. Therefore, these results are made independent of the flow direction if written as:

$|\varphi|=1+\varphi_{0}, \quad$ with $\varphi_{0} \sim 3-e+1.5 k_{i} c_{i n}+O\left(k_{i}^{2}\right) \quad$ as $k_{i} \rightarrow 0$.

However, if significant inhibition is present it is no longer reasonable to assume that $\varphi_{0} \ll<1$. Introducing Eq.(30a) into (36) and considering only the more important terms as $k_{i} \rightarrow \infty$, it is possible to show that $\varphi=k_{i}+O(1)$, or generalizing with respect to the flow direction:

$|\varphi|=k_{i} c_{i n}+O(1)$

as $k_{i} \rightarrow \infty$.

Note that since for inhibited kinetics, $\varphi$ writes as in Eq.(26), then Eq.(44b) actually means that $v \phi^{2} /\left|P_{C}\right|=1$, which is the leading term in the critical condition for concentration annulment from a zero-order reaction, as shown in Eq.(41b).

To describe the complete range of values of the inhibition constant $k_{i}$, both asymptotes in Eq.(44) can be combined in a correlation, resulting in Eq.(48). This is compared with the numerical solution of Eq.(36) using the exact solution in terms of Lambert's function in Fig. 11. 
This correlation was used to predict the location of the optimum enhancement points in Figs. 8, 12 and 13. In general, the same procedure could be applied to kinetics of the form presented in Eq.(2), but it is reasonable to believe that the behavior of $\varphi\left(k_{i}\right)$ will change between the results for reactions of order $m\left(\right.$ as $\left.k_{i} \rightarrow 0\right)$ and order $m-p$ (as $\left.k_{i} \rightarrow \infty\right)$. Therefore, a description based on these asymptotes seems to be simpler, and thus more valuable.

An interpretation of the two regions divided by the line in Fig. 11 can be given, taking into account the results for zero-order kinetics. Thus, for $\varphi$ above that given by Eq.(48) it is possible to anticipate that concentration will attain low values in an appreciable fraction of the membrane volume (which increases with $\varphi$ ). Below this line, kinetically controlled conditions are approached. This expression delimits hypoxic and non-hypoxic conditions in bioreactors with any degree of reactant inhibition.

The maximum enhancement can be calculated by Eq.(34), using the approximations for the effectiveness factor given previously. Figs. 12 and 13 show the enhancement in the reaction rate with Michaelis-Menten kinetics and different degrees of inhibition. The prediction of $\mathrm{E}_{\max }$ by Eq.(48) is very reasonable, improving when $c_{2} \rightarrow 0$ and $\varepsilon \rightarrow 0$. Better estimates would require the knowledge of the higher order term represented by $\varphi_{1}$, but for practical purposes the derived approximations are reasonable. The enhancement increases as $\varepsilon$ increases, but this corresponds to lower effectiveness factors (Fig. 8). The optimum locus for $P=10$ practically coincides with the one for $P=50$, for the same $\varepsilon$ and $c_{2}$.

\subsubsection{Practical example}

Nakajima et al. [61] studied sucrose inversion in forced-flow through ceramic membranes. Complete conversion was observed in a membrane with a support with pore size of $0.5 \mu \mathrm{m}$. The timescales for the processes that occur were estimated by the authors, resulting in the following values for the dimensionless numbers: $P=265.3$ (shortest residence time), $\phi^{2}=961.5$ (with the reaction timescale written for a zero-order reaction), and $\varepsilon=0.25$ (the membrane is cylindrical with $t_{m}=1 \mathrm{~mm}$ ). They also estimate the timescale for radial pore 
diffusion, which when compared with the one for reaction yields $D a_{\text {pore }}=6 \times 10^{-4}$, justifying the pseudo-homogeneous approach in which Eq.(1) is based. The operation is characterized by a "flow-through Damköhler number" equal to $\varphi=4.1$. The critical value of Thiele modulus for this value of $P$ can be calculated according to Eq.(41b). Thus, maximum enhancement occurs at $\varphi=1.2$, and from Eq.(43), $\mathrm{E}_{\max }=11.7$. The enhancement observed at the operating conditions would be lower, which means that the objective of complete conversion is privileged, according to the results in section 5.3.4 (zero-order reactions).

However, the same authors had previously presented [62] a kinetic model accounting for substrate inhibition for the same reaction and enzyme in membranes with different loading. It was written as:

$$
\hat{R}(\hat{c})=\frac{V_{m} \hat{c}}{K_{m}+\hat{c}+\hat{c}^{2} / K_{s}}
$$

We will assume that the same values of $K_{m}$ and $K_{s}$ can be used in the conditions of Nakajima et al. [61], who report $V_{m}=237 \mathrm{~mol} /\left(\mathrm{m}^{3} \mathrm{~s}\right)$. For an inlet concentration of sucrose equal to $\hat{c}_{1}=300 \mathrm{~mol} / \mathrm{m}^{3}, \hat{R}(\hat{c})$ can be written in dimensionless form as

$$
R(c)=\frac{\left(1+k_{i}+k_{i 2}\right) c}{1+k_{i} c+k_{i 2} c^{2}},
$$

where $k_{i}=7.5$ and $k_{i 2}=3.2\left(55^{\circ} \mathrm{C}\right.$ and $\left.\mathrm{pH} 4.7\right)$. Using this kinetic expression, the Thiele modulus given in Eq.(3) is calculated as $\phi^{2}=182.2$. We adapt the membrane Damköhler number for this case, which calculates as:

$$
\varphi=\left(1+k_{i}+k_{i 2}\right) \frac{v \phi^{2}}{P_{C}}=9.1
$$

Comparing with the prediction from Eq.(48) (which, nevertheless, ignores the contribution of $\left.k_{i 2}\right), \varphi=8.9$, it is reasonable to claim that the experimental design and operating conditions that were found by the authors as more advantageous are very close to the theoretical predictions for maximum enhancement. 


\subsubsection{Inward flow}

When $P_{C}<0$, the problem is formulated in similar terms, if $\varphi$ is replaced by $\varphi_{2}$ for power-law kinetics:

$\varphi_{2}=\frac{v}{-P_{C}} \frac{t_{m}^{2}}{D_{\text {eff }}} \frac{R\left(\hat{c}_{2}\right)}{\hat{c}_{2}}=\frac{v}{-P_{C}} \frac{t_{m}^{2}}{D_{\text {eff }}} \hat{c}_{2}^{m-1}=1+\varphi_{0}+\frac{\varphi_{1}}{-P_{C}}$,

where $\varphi_{0}$ and $\varphi_{1}$ are given in Eqs.(47) (Table 3). The result for Michaelis-Menten kinetics (given in Eq.(48)) is generalized regarding the flow direction. The conditions for maximum enhancement are predicted in Fig. 14 for both types of reaction rate laws. The values of $\mathrm{E}_{\max }$ given by Eq.(34) are in very good agreement with the ones obtained numerically. For the second-order reaction, the asymptotic solution in Regime IV for E is calculated from Eqs.(22) and (33b). It is possible to observe that in inward flow, the enhancement decreases as $\varepsilon$ increases, contrary to the behavior in Figs. 10 and 13. In the case of moderate inhibition ( $k_{i}=1$ ), Eq.(29b) was replaced into Eq.(34).

\subsection{Catalytic 'dead end' filtration (Fig. 2B)}

When the concentration leaving the membrane does not change by the action of significant axial sweep flow on the permeate side, the 'no flux' boundary condition at the downstream surface is appropriate. We have noted in a previous work [49] that in this case, the concentration profile cannot present the structure described in section 5.1. However, as we have also suggested, this regime has special significance, as the same results not only predict the maximum enhancement of the effectiveness factor, but also provide an interesting answer to the effectiveness-conversion trade-off that appears in these membrane radial flow reactors.

Numerical results for the enhancement factor in an outward radial flow membrane with nonlinear kinetics are shown in Fig. 15 for moderate and high values of the Peclet number. The asymptotic predictions in each regime were calculated as follows: $(i)$ when $P=1$ the solution in Table 1 for Regime I was used; (ii) the effectiveness factor under strong convective conditions given by Eq.(13) can be compared with the diffusive limit of the solution in Table 1 to yield 
enhancement in Regime II; (iii) the diffusional asymptote of these plots is well represented by Eq.(21) with $c_{2}=0$; (iv) results for Regime IV are obtained as in Eq.(34), but the value of the effectiveness factor under diffusive conditions $\left(K_{\infty} /(v \phi)\right)$ can be replaced by a correlation such as the one in Eq.(22); (v) the condition for maximum enhancement $\left(\varphi_{\text {opt }}\right)$ is predicted from Eqs.(46) in Table 3 and $\mathrm{E}_{\max }\left(\varphi_{\text {opt }}\right)$ follows directly from Eq.(34). It can be seen that the optimum conditions (derived for specified surface concentration downstream) describe very accurately the behavior of these systems, even at moderate values of the Peclet number.

\section{THIELE-PECLET OPERATING DIAGRAM}

Each regime characterized in previous sections corresponds to a limited area in a map with the dimensionless parameters governing the system behavior as axes. We consider that the overall picture containing the several operating possibilities for the catalytic membrane can be mapped onto a $P-\phi^{2}$ diagram, since the other parameters are either fixed for a given geometry ( $\varepsilon$ or $v$ ), reactional system (e.g. $m$ ), or determined in association with the external problems in channel and shell sides (e.g. fixed surface concentrations $\hat{c}_{1}$ and $\hat{c}_{2}$ ). Thus, we will restrict ourselves to this representation (comparing diffusion, viscous permeation and reaction phenomena), noting that the previous analytical results easily allow for other diagrams (including effects from curvature, surface concentration asymmetry or order of reaction) to be plotted, if desired.

Fig. 16 shows the distribution of the effectiveness factor in a flow-through membrane for all combinations of Peclet and Thiele numbers, while Fig. 17 refers to the enhancement factor. In these representations, the transitions between the regimes are given by Eqs.(17), (23), (24) and (39). Apart from numerical factors, the $P-\phi^{2}$ dependence in these expressions is summarized by:

$$
\phi^{*}=\left\{\begin{array}{ll}
O(1), & P \rightarrow 0 \\
\frac{(1+P)^{3}}{P^{2}}, & P \sim 1 \\
P, & P \rightarrow \infty
\end{array} .\right.
$$


The effect of the reaction rate law, membrane curvature and concentration downstream is introduced when considering the full expressions, given previously. The scaling laws in Eq.(51) separate regions where significant mass transfer resistance (concentration decay) inside the membrane exists $\left(\phi>\phi^{*}\right)$ or not $\left(\phi<\phi^{*}\right)$. Regimes I and III (convection controlled) present low enhancement, with the effectiveness factor decreasing from its kinetically controlled diffusive value $\left(\eta_{0}\right)$ to zero, according to the high Thiele modulus asymptote, Eq.(20). Convective dominance in the mass transport yields the maximum effectiveness regime (Regime II), for $P^{*}>O(1)$. Though Regime IV is associated with an intermediate region of $\eta$, maximum enhancement conditions are observed, as predicted by the analysis. Note that $\mathrm{E}_{\max }$ is described analytically assuming that $\phi^{2} / P \sim 1$ (Regime IV, $\phi / P \sim 1 / \phi<<1$ ), which is in good agreement with the coordinates of the minima in the iso-E curves in Fig. 17. On the other hand, the subdominant term in Regime III (e.g. see Eq.(18)) is of order $P / \phi$, which must be small comparing with the leading-order term, of $O(1)$. Thus, the scaling in Eq.(51) at moderate to high Peclet number $(\phi / P \sim 1)$ is confirmed, separating Regime III $(\phi / P>>1)$ from Regime IV $(\phi / P<<1)$.

\section{CONCLUSIONS}

We considered the improvement of mass transfer by promotion of an additional transport mechanism (internal forced convection) in permeable flat or curved catalytic membranes. Using perturbation methods for small and large values of the governing parameters (the Peclet number $P$, and the Thiele modulus $\phi^{2}$ ), we are able to analytically cover the whole spectrum of regimes. These techniques are appropriate since we consider nonlinear reaction rate laws, which make exact solutions unattainable. In particular, we focused on the calculation of the effectiveness factor $(\eta)$ and of the enhancement of $\eta$ which is caused by convection, or perfusion (measured by the factor E). Expressions for $\eta$ and $\mathrm{E}$ are given for four different regimes (I: chemical control under diffusive conditions; II: chemical control under convective 
conditions; III: diffusional control; IV: convective dominated systems) for different modes of operation ('open' or 'dead-end' configurations) and flow directions (outward or inward).

We were also interested in the identification of the conditions which maximize E. It is known from previous work, that the effectiveness factor (or equivalently, the average reaction rate) in a membrane (bio)reactor will always increase with perfusion, but this improvement is only appreciable if the reaction rate is tuned in a very specific manner. In fact, under certain conditions the enhancement may be only residual. Here, we have also derived analytically the relationship that must hold between governing parameters (comparing reaction, diffusion and permeation), so as to take maximum advantage from the catalytic membrane. These conditions are a function of the reaction rate law (e.g. of the order of reaction $m$ ) and determine the location and magnitude of the maximum enhancement in the effectiveness factor. These expressions are in excellent agreement with the numerical solution of the reaction-transport differential equation and provide a simple basis for the design of these reactors. Moreover, we have also considered the effect of reactant inhibition on these conditions, which is essential to analyze the increasing number of biotechnological applications. The main observations are that: (i) the optimum Thiele and Peclet numbers should be related by $\phi^{2} \simeq\left(1+\varphi_{0}\right) P$, where $\varphi_{0}$ is a given function of the order of reaction; (ii) the optimum value of $\phi^{2} / P$ as a function of the dimensionless inhibition constant separates two regions, one where appreciable reactant exhaustion occurs (i.e. hypoxic conditions in bioreactors), from another where kinetically controlled conditions are attained; (iii) the previous observation is found to be exact for a zeroorder reaction, where a critical value of the Thiele modulus $\left(\phi_{C}^{2} \simeq P+\ln (P)+1\right.$ for strong convective conditions) separates the two asymptotes of the effectiveness factor ( $\eta=1$ for $\phi^{2}<\phi_{C}^{2}$ and $\eta=P / \phi^{2}$ for $\phi^{2}>\phi_{C}^{2}$ ); (iv) the maximum enhancement to be expected is proportional to the conversion that would be observed in a pseudo-homogeneous plug-flow reactor without diffusion, operated at the optimum values of Thiele (which replaces Damköhler) and Peclet numbers; $(v)$ the generic dependence already found by the authors for $\mathrm{E}_{\max }$ (written as $\mathrm{E}_{\max } \sim \phi \sim \sqrt{P}$ ) is verified, but the numerical coefficient which makes the rule quantitative is 
a non-negligible function of the form of the reaction rate law (given here explicitly); (vi) the situation of inward flow is described by minor modifications, and in some cases the results can be normalized with respect to the flow direction; (viii) surprisingly, even though the structure of the concentration profile in 'optimum conditions' is not consistent in the case of 'dead-end' operation, the enhancement $\mathrm{E}$ is very well described by the solutions derived; (ix) for values of $P$ below a given limit (of order $O(1)$ ) and when a concentration difference exists between both surfaces, the maximum enhancement may be observed at $\phi^{2} \rightarrow 0$, which is however lower than the one observed at high Thiele and Peclet numbers.

We emphasize that this regime of maximum enhancement may be highly interesting (even though moderate values of the effectiveness factor can prevail): (i) when maximum $\eta$ is obtained under kinetically controlled conditions, which may be undesirable in practice, since this will require longer membranes to attain a given conversion (with higher investment cost, reactor volume, and axial pressure drop associated); (ii) when $\eta$ is already relatively high or intermediate, the conditions under which these reactors are competitive with other technologies must be well identified; (iii) in bioreactors where strong perfusion can be the only strategy to achieve conditions of homogeneous growth, avoiding the presence of 'dead cores'. Actually, in this latter context, the factor $\mathrm{E}$ could be thought of as the fractional increase in the 'active' length of the bioreactor, compared with the one that develops when nutrients are supplied by diffusion alone.

The consideration of asymmetrical boundary conditions also led to the appearance of several interesting results, which have not been identified in previous literature, even in the case of the diffusion-reaction problem. This analysis has several implications in hollow fiber bioreactors, catalysts exposed at non-uniform conditions, etc. It is particularly relevant to occur in the operation of a membrane reactor. The following results in the diffusive limit were obtained: $(i)$ the maximum effectiveness factor $\eta_{0}$ (observed in the chemical regime) decreases when the concentration established at the reference surface increases compared to the one at the other surface, (ii) this decay of $\eta_{0}$ is more pronounced for order of reaction above 1 and for thicker membranes, (iii) $\eta_{0}$ depends on the form of the reaction rate law (noting that for 
symmetric boundary conditions, it always equals 1), (iv) the asymptotic behavior of the effectiveness factor at high Thiele modulus includes contributions from two non-symmetrical boundary layers, which generalizes our previous approximation [53], and (v) a correlation including kinetic and diffusional limits is provided, describing reasonably well the intermediate range of $\phi^{2}$.

Finally, the transition between the different limiting cases is superimposed with the distribution of effectiveness and enhancement factors in Peclet-Thiele maps. Therefore, the overall picture as well as extensive characterization of all relevant operating regimes is attained.

\section{ACKNOWLEDGEMENTS}

J. P. Lopes gratefully acknowledges financial support from FCT - Fundação para a Ciência e a Tecnologia (SFRH/BPD/81532/2011).

\section{NOTATION}

a characteristic distance for diffusion in the membrane lumen, $m$

$\hat{c} \quad$ concentration of reactant in the membrane, $\mathrm{mol} / \mathrm{m}^{3}$

$c$ dimensionless concentration of reactant in the membrane, $=\hat{c} / \hat{c}_{1}$

$\langle c\rangle \quad$ radially-averaged reactant concentration in the membrane

$\left\langle c_{0}\right\rangle \quad$ leading-order radially-averaged reactant concentration in the membrane in Eq.(6a)

$\left\langle c_{0}^{D}\right\rangle$ leading-order radially-averaged reactant concentration in the diffusive membrane in Eq.(6b)

$\hat{c}_{1} \quad$ dimensional surface concentration at $r=0, \mathrm{~mol} / \mathrm{m}^{3}$

$\hat{c}_{2} \quad$ dimensional surface concentration at $r=1, \mathrm{~mol} / \mathrm{m}^{3}$

$c_{2} \quad$ dimensionless ratio between concentrations at the surfaces, $=\hat{c}_{2} / \hat{c}_{1}$

$c_{\text {in }}$ dimensionless concentration at the surface through which flow enters the membrane

$D_{\text {eff }} \quad$ effective diffusivity in the membrane (transverse), $\mathrm{m}^{2} / \mathrm{s}$

$D a_{\text {pore }}$ Damköhler number at the pore scale (with pore diffusion timescale) 
E enhancement factor given by Eq.(5)

$k \quad$ reaction kinetic constant, $1 / \mathrm{s}$

$k_{i} \quad$ dimensionless inhibition constant

$k_{i}^{*} \quad$ inhibition constant, $\mathrm{m}^{3} / \mathrm{mol}$

$K_{\infty}, \tilde{K}_{\infty}$ kinetic factors in the diffusional regime

$L \quad$ length of the channel, m

LHS left hand side

$m \quad$ order of reaction

$p \quad$ kinetic parameter in Eq.(2)

$P \quad$ internal (radial) Peclet number, $P=V\langle\hat{u}\rangle /\left(S D_{\text {eff }}\right)$

$P_{C} \quad$ internal Peclet number including curvature effects, $=P-\varepsilon \sigma$

$\hat{p}_{\text {in }} \quad$ pressure on the lumen side, $\mathrm{Pa}$

$\hat{p}_{\text {out }} \quad$ pressure on the extracapillary side, $\mathrm{Pa}$

$r \quad$ dimensionless transverse coordinate

$\hat{R} \quad$ reaction rate per membrane volume, $\mathrm{mol} /\left(\mathrm{m}^{3} . \mathrm{s}\right)$

$R \quad$ dimensionless reaction rate, Eq.(2)

RHS right hand side

$S \quad$ area of the membrane-lumen interface, $\mathrm{m}^{2}$

$t_{m} \quad$ thickness of the catalytic coating, $\mathrm{m}$

$\hat{u} \quad$ fluid (radial) superficial velocity, $\mathrm{m} / \mathrm{s}$

$u \quad$ dimensionless fluid (radial) superficial velocity

$\langle\hat{u}\rangle \quad$ radially averaged superficial fluid velocity, $\mathrm{m} / \mathrm{s}$

$V \quad$ membrane volume, $\mathrm{m}^{3}$

$X \quad$ reactant conversion

$W(x) \quad$ Lambert-W function or product logarithm function

$z \quad$ dimensionless axial coordinate, $\hat{z} / L$ 


\section{Greek letters}

$\alpha \quad$ aspect ratio of the membrane

$\varepsilon \quad$ ratio of the characteristic distances for diffusion in the membrane and in the lumen

$\phi^{2} \quad$ Thiele modulus

$\phi_{C}^{2} \quad$ critical value of the Thiele modulus for a zero-order reaction

$\hat{\delta} \quad$ thickness of the boundary layer

$\Gamma(a, b)$ incomplete Gamma function

$\eta \quad$ effectiveness factor

$\eta_{0} \quad$ effectiveness factor in the chemical regime under diffusive conditions

$\eta_{\infty} \quad$ effectiveness factor in the diffusional regime under diffusive conditions

$\varphi \quad$ flow-through membrane Damköhler number, $=\left(1+k_{i}\right)^{p} v \phi^{2} / P_{C}$

$\varphi_{2} \quad$ flow-through membrane Damköhler number referred to the outer surface conditions for inward flow

$\mu \quad$ dynamic viscosity, Pa.s

$v \quad$ volume to surface ratio, divided by characteristic dimension for diffusion

$\Lambda \quad$ shape factors

Superscripts

$\wedge$ dimensional quantities
D diffusive conditions (absent viscous flow)

Subscripts

in inner region (boundary layer) in the convection-reaction dominated regime

$\max \quad$ maximum

min minimum

opt optimum

out outer region in the convection-reaction dominated regime

wall lumen-membrane interface 


\section{REFERENCES}

[1] A. Nir, L.M. Pismen, Simultaneous intraparticle forced convection, diffusion and reaction in a porous catalyst, Chemical Engineering Science, 32 (1977) 35-41.

[2] A. Rodrigues, B. Ahn, A. Zoulalian, Intraparticle Forced Convection Effect in Catalyst Diffusivity Measurements and Reactor Design, AIChE Journal, 28 (1982) 541-546.

[3] R. Cogan, G. Pipko, A. Nir, Simultaneous Intraparticle Forced Convection, Diffusion and Reaction in a Porous Catalyst - 3, Chemical Engineering Science, 37 (1982) 147-151.

[4] S. Cheng, A. Zoulalian, J.P. Brunelle, Influence of the porous structure of alumina pellets and the internal convective flow on the effective diffusivity of exhaust gas catalyst, in: A. Crucq, A. Frennet (Eds.) Catalysis and Automative Pollution Control, Elsevier Science Publishers B.V., Amsterdam, 1987, pp. 323-332.

[5] C.L. Prince, V. Bringi, M.L. Shuler, Convective Mass Transfer in Large Porous Biocatalysts: Plant Organ Cultures, Biotechnology Progress, 7 (1991) 195-199.

[6] E.L.G. Oliveira, C.A. Grande, A.E. Rodrigues, Methane steam reforming in large pore catalyst, Chemical Engineering Science, 65 (2010) 1539-1550.

[7] E.L.G. Oliveira, C.A. Grande, A.E. Rodrigues, Effect of catalyst activity in SMR-SERP for hydrogen production: Commercial vs. large-pore catalyst, Chemical Engineering Science, 66 (2011) 342-354.

[8] A.E. Rodrigues, Permeable packings and perfusion chromatography in protein separation, Journal of Chromatography B: Biomedical Applications, 699 (1997) 47-61.

[9] A.E. Rodrigues, J.M. Loureiro, C. Chenou, M. Rendueles de la Vega, Bioseparations with permeable particles, Journal of Chromatography B: Biomedical Applications, 664 (1995) 233240.

[10] M.W. Young, R.C. Dean, Optimization of Mammalian-Cell Bioreactors, Bio-Technology, 5 (1987) 835-837.

[11] K. Breitenbucher, M. Siegl, A. Knupfer, M. Radke, Open-pore sintered glass as a highefficiency support medium in bioreactors: New results and long-term experiences achieved in high-rate anaerobic digestion, Water Science and Technology, 22 (1990) 25-32.

[12] J.A. Schonberg, G. Belfort, Enhanced Nutrient Transport in Hollow Fiber Perfusion Bioreactors: A Theoretical Analysis, Biotechnology Progress, 3 (1987) 80-89.

[13] M. Devarapalli, B.J. Lawrence, S.V. Madihally, Modeling nutrient consumptions in large flow-through bioreactors for tissue engineering, Biotechnology and Bioengineering, 103 (2009) 1003-1015.

[14] P.D. Hay, A.R. Veitch, J.D.S. Gaylor, Oxygen transfer in a convection-enhanced hollow fiber bioartificial liver, Artificial Organs, 25 (2001) 119-130.

[15] Y. Huang, Z. Zhou, Y. Qi, X. Li, Z. Cheng, W. Yuan, Hierarchically macro-/mesoporous structured Co-Mo-Ni/ $\gamma$-Al $2 \mathrm{O} 3$ catalyst for the hydrodesulfurization of thiophene, Chemical Engineering Journal, 172 (2011) 444-451.

[16] K.M. De Lathouder, J. Bakker, M.T. Kreutzer, F. Kapteijn, J.A. Moulijn, S.A. Wallin, Structured reactors for enzyme immobilization: Advantages of tuning the wall morphology, Chemical Engineering Science, 59 (2004) 5027-5033.

[17] A.A. Khassin, T.M. Yurieva, A.G. Sipatrov, V.A. Kirillov, G.K. Chermashentseva, V.N. Parmon, Fischer-Tropsch synthesis using a porous catalyst packing: Experimental evidence of an efficient use of permeable composite monoliths as a novel type of the Fischer-Tropsch synthesis catalyst, Catalysis Today, 79-80 (2003) 465-470.

[18] H.C. Aran, J.K. Chinthaginjala, R. Groote, T. Roelofs, L. Lefferts, M. Wessling, R.G.H. Lammertink, Porous ceramic mesoreactors: A new approach for gas-liquid contacting in multiphase microreaction technology, Chemical Engineering Journal, 169 (2011) 239-246.

[19] J.M. Jani, H. Can Aran, M. Wessling, R.G.H. Lammertink, Modeling of gas-liquid reactions in porous membrane microreactors, Journal of Membrane Science, 419-420 (2012) 5764.

[20] H.C. Aran, H. Klooster, J.M. Jani, M. Wessling, L. Lefferts, R.G.H. Lammertink, Influence of geometrical and operational parameters on the performance of porous catalytic membrane reactors, Chemical Engineering Journal, 207-208 (2012) 814-821. 
[21] P.A. Kumar, M.D. Tanwar, S. Bensaid, N. Russo, D. Fino, Soot combustion improvement in diesel particulate filters catalyzed with ceria nanofibers, Chemical Engineering Journal, 207208 (2012) 258-266.

[22] H. Ström, S. Sasic, B. Andersson, Design of automotive flow-through catalysts with optimized soot trapping capability, Chemical Engineering Journal, 165 (2010) 934-945.

[23] S.R.A. de Loos, J. van der Schaaf, M.H.J.M. de Croon, T.A. Nijhuis, J.C. Schouten, Heterogeneous catalysis in a microchannel using a layer of carbon nanofibers on the channel wall, Chemical Engineering Journal, 179 (2012) 242-252.

[24] L.J. Kelsey, M.R. Pillarella, A.L. Zydney, Theoretical analysis of convective flow profiles in a hollow-fiber membrane bioreactor, Chemical Engineering Science, 45 (1990) 3211-3220.

[25] W.S. Long, S. Bhatia, A. Kamaruddin, Modeling and simulation of enzymatic membrane reactor for kinetic resolution of ibuprofen ester, Journal of Membrane Science, 219 (2003) 6988.

[26] M.M.Y. Motamedhashemi, F. Egolfopoulos, T. Tsotsis, Application of a flow-through catalytic membrane reactor (FTCMR) for the destruction of a chemical warfare simulant, Journal of Membrane Science, 376 (2011) 119-131.

[27] T. Westermann, T. Melin, Flow-through catalytic membrane reactors-Principles and applications, Chemical Engineering and Processing: Process Intensification, 48 (2009) 17-28.

[28] R. Dittmeyer, V. Höllein, K. Daub, Membrane reactors for hydrogenation and dehydrogenation processes based on supported palladium, Journal of Molecular Catalysis A: Chemical, 173 (2001) 135-184.

[29] J.G. Sanchez Marcano, T.T. Tsotsis, Catalytic Membranes and Membrane Reactors, WileyVCH Verlag GmbH \& Co. KGaA, 2004.

[30] I. Coelhoso, J. Crespo, A.E. Rodrigues, The effect of convective flow on the facilitated transport across a membrane, in: Meeting of the European Membrane Society, Paris, 1992.

[31] D. Fritsch, I. Randjelovic, F. Keil, Application of a forced-flow catalytic membrane reactor for the dimerisation of isobutene, Catalysis Today, 98 (2004) 295-308.

[32] B. Golman, K. Shinohara, M. Kobayashi, Selectivity and yield of exothermic consecutive reactions in catalytically active porous membrane reactor, Journal of Chemical Engineering of Japan, 30 (1997) 507-513.

[33] M. Kobayashi, T. Kanno, J.I. Horiuchi, S. Hoshi, N. Hattori, J. Togawa, Hysteresis kinetics of propene oxidation characterized by a Ag-Re supported membrane reactor, in, 2001, pp. 105112.

[34] M. Kobayashi, J. Togawa, T. Kanno, J.I. Horiuchi, K. Tada, Dramatic innovation of propene epoxidation efficiency derived from a forced flow membrane reactor, Journal of Chemical Technology and Biotechnology, 78 (2003) 303-307.

[35] A. Schmidt, R. Haidar, R. Schomäcker, Selectivity of partial hydrogenation reactions performed in a pore-through-flow catalytic membrane reactor, Catalysis Today, 104 (2005) 305312.

[36] J. Coronas, J. Santamaría, Catalytic reactors based on porous ceramic membranes, Catalysis Today, 51 (1999) 377-389.

[37] M. Reif, R. Dittmeyer, Porous, catalytically active ceramic membranes for gas-liquid reactions: A comparison between catalytic diffuser and forced through flow concept, Catalysis Today, 82 (2003) 3-14.

[38] S. Bénard, A. Giroir-Fendler, P. Vernoux, N. Guilhaume, K. Fiaty, Comparing monolithic and membrane reactors in catalytic oxidation of propene and toluene in excess of oxygen, Catalysis Today, 156 (2010) 301-305.

[39] A. Schmidt, A. Wolf, R. Warsitz, R. Dittmeyer, D. Urbanczyk, I. Voigt, G. Fischer, R. Schomäcker, A pore-flow-through membrane reactor for partial hydrogenation of 1,5cyclooctadiene, AIChE Journal, 54 (2008) 258-268.

[40] D. Urbanczyk, R. Dittmeyer, A. Wolf, R. Warsitz, G. Fischer, I. Voigt, Evaluation of porous catalytic membranes operated in pore-flow-through mode for hydrogenation of $\alpha$ methylstyrene, Asia-Pacific Journal of Chemical Engineering, 5 (2010) 12-25.

[41] M. Pera-Titus, M. Fridmann, N. Guilhaume, K. Fiaty, Modelling nitrate reduction in a flow-through catalytic membrane contactor: Role of pore confining effects on water viscosity, Journal of Membrane Science, 401-402 (2012) 204-216. 
[42] H.J. Sloot, G.F. Versteeg, W.P.M. Van Swaaij, A non-permselective membrane reactor for chemical processes normally requiring strict stoichiometric feed rates of reactants, Chemical Engineering Science, 45 (1990) 2415-2421.

[43] G. Saracco, J.W. Veldsink, G.F. Versteeg, W.P.M. van Swaaij, Catalytic combustion of propane in a membrane reactor with separate feed of reactants-II. Operation in presence of trans-membrane pressure gradients, Chemical Engineering Science, 50 (1995) 2833-2841.

[44] M. Murru, A. Gavriilidis, Catalytic combustion of methane in non-permselective membrane reactors with separate reactant feeds, Chemical Engineering Journal, 100 (2004) 2332.

[45] J. Xuan, D.Y.C. Leung, M.K.H. Leung, M. Ni, H. Wang, Chemical and transport behaviors in a microfluidic reformer with catalytic-support membrane for efficient hydrogen production and purification, International Journal of Hydrogen Energy, 37 (2012) 2614-2622.

[46] M. Zahorodny-Burke, B. Nearingburg, A.L. Elias, Finite element analysis of oxygen transport in microfluidic cell culture devices with varying channel architectures, perfusion rates, and materials, Chemical Engineering Science, 66 (2011) 6244-6253.

[47] Y.H. Hsu, M.L. Moya, P. Abiri, C.C.W. Hughes, S.C. George, A.P. Lee, Full range physiological mass transport control in 3D tissue cultures, Lab on a Chip - Miniaturisation for Chemistry and Biology, 13 (2013) 81-89.

[48] C. Kim, K. Kreppenhofer, J. Kashef, D. Gradl, D. Herrmann, M. Schneider, R. Ahrens, A. Guber, D. Wedlich, Diffusion- and convection-based activation of Wnt/ $\beta$-catenin signaling in a gradient generating microfluidic chip, Lab on a Chip - Miniaturisation for Chemistry and Biology, 12 (2012) 5186-5194.

[49] J.P. Lopes, M.A. Alves, M.N. Oliveira, S.S. Cardoso, A.E. Rodrigues, Internal mass transfer enhancement in flow-through catalytic membranes, under review, (2013).

[50] E. Nagy, Mass transfer through a convection flow catalytic membrane layer with dispersed nanometer-sized catalyst, Industrial and Engineering Chemistry Research, 49 (2010) 10571062.

[51] J.W. Bjork, R.T. Tranquillo, Transmural flow bioreactor for vascular tissue engineering, Biotechnology and Bioengineering, 104 (2009) 1197-1206.

[52] J.P. Lopes, S.S.S. Cardoso, A.E. Rodrigues, Convection, diffusion, and exothermic ZeroOrder reaction in a porous catalyst slab: Scaling and perturbation analysis, AIChE Journal, 55 (2009) 2686-2699.

[53] J.P. Lopes, S.S.S. Cardoso, A.E. Rodrigues, Effectiveness factor for thin catalytic coatings: Improved analytical approximation using perturbation techniques, Chemical Engineering Science, 71 (2012) 46-55.

[54] C.S. Tan, J.M. Smith, Catalyst particle effectiveness with unsymmetrical boundary conditions, Chemical Engineering Science, 35 (1980) 1601-1609.

[55] J.A. DeSimone, J.A. Pennline, A new asymptotic analysis of the nth order reactiondiffusion problem: Analytical and numerical studies, Mathematical Biosciences, 40 (1978) 303318.

[56] M. Chaara, R. Noble, Effect of Convective Flow Across a Film on Facilitated Transport, Separation Science and Technology, 24 (1989) 893-903.

[57] R. Aris, The mathematical theory of diffusion and reaction in permeable catalysts, Oxford University Press, London, 1975.

[58] C.M. Bender, S.A. Orszag, Advanced Mathematical Methods for Scientists and Engineers, McGraw-Hill, New York, 1978.

[59] A.E. Rodrigues, J.M. Orfao, A. Zoulalian, Intraparticle convection, diffusion and zero order reaction in porous catalysts, Chemical Engineering Communications, 27 (1984) 327-337.

[60] G. Stephanopoulos, K. Tsiveriotis, Effect of intraparticle convection on nutrient transport in porous biological pellets, Chemical Engineering Science, 44 (1989) 2031-2039.

[61] M. Nakajima, A. Watanabe, N. Jimbo, K. Nishizawa, S.-i. Nakao, Forced-flow bioreactor for sucrose inversion using ceramic membrane activated by silanization, Biotechnology and Bioengineering, 33 (1989) 856-861.

[62] M. Nakajima, N. Jimbo, K. Nishizawa, H. Nabetani, A. Watanabe, Conversion of sucrose by immobilized invertase in an asymmetric membrane reactor, Process Biochemistry, 23 (1988) 32-35. 
[63] G.F. Froment, K.B. Bischoff, Chemical Reactor Analysis and Design Wiley, New York, 1979.

[64] J.C. Gottifredi, E.E. Gonzo, O.D. Quiroga, Isothermal effectiveness factor-I. Analytical expression for single reaction with arbitrary kinetics. Slab geometry, Chemical Engineering Science, 36 (1981) 713-719.

[65] J.C. Gottifredi, E.E. Gonzo, O.D. Quiroga, Isothermal effectiveness factor-II. Analytical expression for single reaction with arbitrary kinetics, geometry and activity distribution, Chemical Engineering Science, 36 (1981) 721-730. 


\section{APPENDiX A}

\section{CHEMICAL REGIME IN A DIFFUSIVE CATALYTIC MEMBRANE}

I. Maximum effectiveness factor for a diffusive membrane with arbitrary kinetics and asymmetrical boundary conditions (derivation of Eq.(9))

The maximum effectiveness factor attainable in an isothermal membrane with 'normal' kinetics $\left(d \eta / d \phi^{2} \leq 0\right)$ and where transport between the two surfaces (kept at distinct concentrations) occurs solely by diffusion does not seem to have been reported previously. In this appendix, we derive this result, previously presented as Eq.(9).

The solution for the effectiveness factor of a cylindrical membrane, with inner and outer walls exposed at specified surface concentrations ( $c=1$ and $c_{2}$, respectively), can be obtained for generic kinetics in the limit of a slow reaction. The inert concentration profile is given by

$c_{0}^{D}(r)=1-\left(1-c_{2}\right) \frac{\ln (1+\varepsilon r)}{\ln (1+\varepsilon)}$.

The next term in the series expansion for concentration at small Thiele modulus $\left(c^{D}(r)=c_{0}^{D}(r)+c_{1}^{D}(r) \phi^{2}+\ldots\right)$ is given by

$c_{1}^{D}(r)=A\left(1+u_{1}\right)+\frac{B}{\varepsilon} \ln (1+\varepsilon r)\left(1+u_{2}\right)$,

where, $A$ and $B$ are integration constants that must be chosen to satisfy the specified concentrations at the surface, and $u_{1}$ and $u_{2}$ are functions which can be calculated for arbitrary kinetics $R(c)$ from:

$$
\begin{aligned}
& u_{1}=\frac{[\ln (1+\varepsilon)]^{2}}{A \varepsilon^{2}\left(1-c_{2}\right)^{2}} \int R\left(c_{0}^{D}\right)(1+\varepsilon)^{2 \frac{1-c_{0}^{D}}{1-c_{2}}}\left(1-c_{0}^{D}\right) d c_{0}^{D} \\
& u_{2}=\frac{\ln (1+\varepsilon)}{B \varepsilon\left(c_{2}-1\right)} \int R\left(c_{0}^{D}\right)(1+\varepsilon)^{2 \frac{1-c_{0}^{D}}{1-c_{2}}} d c_{0}^{D}
\end{aligned}
$$


From these results, the value of the chemical regime branch of the effectiveness factor $\left(\eta_{0}\right)$ can be calculated from Eq.(4) (for $P=0$ ). For power-law kinetics, solving these equations yields the result in Eq.(9). This corresponds to the value of effectiveness in the chemical regime (noted by $\eta_{0}$, since it is observed as $\phi^{2} \rightarrow 0$ ). As discussed previously, it is a function of the kinetic parameters (namely, the order of reaction $m$ ).

\section{Criterion for internal mass transfer limitation}

For thick curved membranes and nonlinear kinetics with $m>1, \eta_{0}$ is lower than the value for a first-order reaction in a planar membrane with the same ratio between surface concentrations. It may be important to use the correct value of the effectiveness factor in the cases where this quantity is used as a criterion to ensure the absence of internal mass transfer effects (e.g. when measuring intrinsic kinetics). In the chemical reaction engineering literature (see e.g. [63]), observable quantities, such as the Weisz-Prater criterion, are used for this purpose:

$\eta \phi^{2}=\frac{t_{m}^{2} \hat{R}_{o b s}}{D_{\text {eff }} \hat{c}_{1}}$

Then it is reasoned that at low $\phi^{2}, \eta \phi^{2}<<1$, since for a catalyst with uniform surface concentration $(\eta \rightarrow 1)$. In the case of a membrane exposed at two different values of concentration, $\eta_{0}$ may be actually one-order of magnitude lower (see Fig. 3). So, care must be taken if the criterion in Eq.(A.5) is only barely fulfilled.

Another criterion is commonly obtained by allowing a small deviation of the effectiveness factor from 1 , e.g. $|1-\eta|<0.05$, and then taking advantage of the perturbation expansion for small Thiele modulus $[64,65]$. In this case, this criterion must obviously be rewritten as $\left|\eta_{0}-\eta\right| \leq 0.05$. Then, the perturbation series in Eq.(8) for $P \rightarrow 0$ allow us to write:

$\eta_{0}-\eta=R\left(\left\langle c_{0}^{D}\right\rangle\right) R^{\prime}\left(\left\langle c_{0}^{D}\right\rangle\right) \Lambda_{2} \phi^{2}<0.05 \eta_{0}$

where $\left\langle c_{0}^{D}\right\rangle$ and $\Lambda_{2}$ where given in Eqs.(6b) and (11), respectively. Note that the value of $\Lambda_{2}$ when $\varepsilon \rightarrow 0$ (slab limit) is $1 / 12$, which differs from the geometric factor that appears in the 
analysis for a slab catalyst exposed at uniform surface concentration (1/3). This is obviously due to the change in the characteristic dimension for diffusion ( $t_{m}$ vs. $\left.t_{m} / 2\right)$ in the definition of the Thiele modulus. In terms of observables, Eq.(A.6) is given by:

$\eta \phi^{2}<\frac{0.05 \eta_{0}}{R^{\prime}\left(\left\langle c_{0}^{D}\right\rangle\right) \Lambda_{2}}$

The case where $\eta_{0}<1$ is of particular importance in membrane reactors, since the concentration difference at both surfaces is the only effect causing the chemical regime asymptote to deviate from 1 (intraparticular convection, nonlinear kinetics, and nonisothermal effects, all lead to $\eta=1$, as long as $c_{2}=1$ ). Eq.(A.7) is plotted in Fig. A.1. 


\section{FIGURE CAPTIONS}

Fig. 1: Schematic representation of a cylindrical flow-through membrane with outward flow. Radial flow through the porous media occurs with a velocity field, $u(r)$, due to the existence of a transmembrane pressure gradient $\left(\hat{p}_{\text {in }}-\hat{p}_{\text {out }}\right)$. Mass transfer proceeds also by diffusion between surface concentrations at $r=0$ and $r=1\left(\hat{c}_{1}\right.$ and $\hat{c}_{2}$, respectively).

Fig. 2: Operating modes of the flow-through (bio) reactor. Configurations differ on the main direction of the feed stream relative to the orientation of the membrane surface. (A) Axially dominated flow (perfusive membrane). (B) Radially dominated flow (catalytic dead-end filtration).

Fig. 3: Effectiveness of a diffusive membrane in kinetically controlled conditions as a function of the ratio between the concentration at the two surfaces. Values for 3 orders of reaction $m$ and 2 values of relative thickness $\varepsilon$ are considered.

Fig. 4: Effectiveness factor for systems with weak to moderate convection (Regimes I and III) and nonlinear kinetics $(m=2)$ in a thin $(\varepsilon=0.1)$ membrane. Negligible reactant concentration on the permeate-membrane interface ( $\left.c_{2}=0\right)$. Asymptotic behavior described by Eq.(8) in Regime I and by Eq.(18) in Regime III.

Fig. 5: Enhancement (E) as a function of internal Peclet number for low Thiele modulus. Plots for $m=2$ with two values of $c_{2}$ : (a) $\varepsilon=0.1$ and (b) $\varepsilon=1$. (c) Kinetics with $m<1, \varepsilon=0.1$ and $c_{2}=0$. Asymptotic expressions given by Eq.(12) for Regime I and by Eq.(14) for Regime II. Correlation (15) is also shown. Further increase of E with $\phi^{2}$ occurs only when (16) is satisfied. The delimitation of the two regimes is based on Eq.(17). 
Fig. 6: Effectiveness factor for a diffusive membrane with nonlinear kinetics and asymmetric boundary conditions. Correlation: Eq.(22) with $n=0.8$. Asymptotic behaviors: Eqs.(9) and (20).

Fig. 7: Enhancement factor in the slow to moderately intense convection regime for nonlinear kinetics (non-negligible diffusive contribution to the overall transport). In the kinetic regime, the asymptotic behavior of the low Peclet range ( $P=0.1$ and 0.5 ) is given by Eq.(12), while in the intermediate range ( $P=1$ and 5) are calculated by the correlation in (15). Asymptotic behavior in regime III given by (21). The regime transition is predicted by Eq.(23). Parameters: $m=2 ; \varepsilon=0.1$; and $c_{2}=0$

Fig. 8: Effectiveness factor for a membrane with Michaelis-Menten kinetics: (a) weak inhibition, and (b) moderate inhibition. Numerical calculations compared with asymptotic results given by Eqs.(33) (after substitution with Eq.(29)) for Regime IV and (13) for Regime II. Optimum enhancement predicted by (48).

Fig. 9: Enhancement in the effectiveness of a membrane with $\varepsilon=0.1, c_{2}=0$ and a secondorder reaction, for high values of the Peclet number. Numerical solutions (full lines) are compared with the asymptotic predictions for each regime (dashed lines). The conditions for maximum enhancement are identified (optimum $\varphi$ from Eqs.(46) in Table 3).

Fig. 10: Effectiveness enhancement (E) in a catalytic membrane where a second-order reaction occurs, at high Peclet number $(P=100)$. Numerical and asymptotic results for $\mathrm{E}$ and $\mathrm{E}_{\max }$ are compared for two values of $\varepsilon$ and $c_{2}$.

Fig. 11: Optimum value of the Damköhler number for a flow-through membrane as a function of the Michaelis-Menten inhibition constant. The numerical solution of the nonlinear equation defined by (36) is compared with the approximate analytical estimate given by Eq.(48). Representation for $\varepsilon=0.1$ and $c_{2}=0$ in the high $P_{C}$ limit of outward flow. 
Fig. 12: Enhancement plot for a thin membrane $(\varepsilon=0.1)$ at high Peclet number, with weakly inhibited Michaelis-Menten kinetics $\left(k_{i}=0.1\right)$. Maximum enhancement predicted by Eq.(48).

Fig. 13: Enhancement plot at high Peclet number for Michaelis-Menten kinetics with moderate inhibition $\left(k_{i}=1\right)$. Maximum enhancement lines as a function of Thiele modulus, and optimal enhancement points are predicted by Eq.(48).

Fig. 14: Maximum enhancement in inward flow $\left(c_{2}=2\right.$ and $\left.P=-50\right)$ for power-law and Michaelis-Menten kinetics with moderate inhibition $\left(k_{i}=1\right)$.

Fig. 15: Enhancement in catalytic 'dead-end' filtration in membrane reactors with (a) weak to moderate convective effects and (b) strong convective effects. Numerical results shown for $m=1.5, \varepsilon=0.1$ and outward flow. Asymptotic predictions in each regime and conditions of maximum enhancement from Eq.(34) are also represented.

Fig. 16: Contours of membrane effectiveness in a Peclet-Thiele diagram. The transitions between the 4 regimes are shown as well as several iso- $\eta$ curves for $m=2 ; \varepsilon=0$ and $c_{2}=0$.

Fig. 17: Enhancement of the membrane effectiveness in a Peclet-Thiele diagram. Contours and iso-E curves are plotted and the parametric areas of the 4 regimes studied are delimitated. Conditions: second-order reaction; thin membrane ( $\varepsilon=0)$, low concentration downstream $\left(c_{2}=0\right)$.

Fig. A.1: Variation of the observable Weisz-Prater criterion for excluding internal mass transfer effects with the concentration ratio, according to Eq.(A.7).

\section{TABLES}


Table 1: Catalytic dead-end filtration in Regime I (Diffusive kinetically controlled limit).

Table 2: Convection-reaction dominated exit concentration for several kinetics.

Table 3: Conditions for maximum effectiveness enhancement in Regime IV. 


\section{TABLES}

Table 1: Catalytic dead-end filtration in Regime I (Diffusive kinetically controlled limit)

\begin{tabular}{|c|c|c|}
\hline $\begin{array}{l}\text { Effectiveness } \\
\text { Factor }\end{array}$ & \multicolumn{2}{|c|}{$\eta=R\left(\left\langle c_{0}\right\rangle\right)+R\left(\left\langle c_{0}\right\rangle\right) R^{\prime}\left(\left\langle c_{0}\right\rangle\right)\left(\Lambda_{1}+\Lambda_{2}|P|\right) \phi^{2}+O\left(\phi^{2} \varepsilon^{2}\right)$} \\
\hline $\begin{array}{c}\text { Enhancement } \\
\text { Factor }\end{array}$ & \multicolumn{2}{|c|}{$\mathrm{E}=1+\frac{\Lambda_{2}|P| \phi^{2} / R\left(\left\langle c_{0}\right\rangle\right)}{1+R^{\prime}\left(\left\langle c_{0}\right\rangle\right) \Lambda_{1} \phi^{2}}$} \\
\hline $\begin{array}{c}\text { Flow } \\
\text { direction }\end{array}$ & Outward & Inward \\
\hline$\left\langle c_{0}\right\rangle$ & 1 & $c_{2}$ \\
\hline \multirow{2}{*}{$\Lambda_{1}$} & $\varepsilon(2+\varepsilon)\left(2+6 \varepsilon+3 \varepsilon^{2}\right)-4(1+\varepsilon)^{4} \ln (1+\varepsilon)$ & $\varepsilon(2+\varepsilon)\left(2-2 \varepsilon-\varepsilon^{2}\right)-4 \ln (1+\varepsilon)$ \\
\hline & $8(2+\varepsilon) \varepsilon^{3}$ & $8(2+\varepsilon) \varepsilon^{3}$ \\
\hline \multirow{2}{*}{$\Lambda_{2}$} & {$\left[\varepsilon(2+\varepsilon)-2(1+\varepsilon)^{2} \ln (1+\varepsilon)\right]^{2}$} & {$[\varepsilon(2+\varepsilon)-2 \ln (1+\varepsilon)]^{2}$} \\
\hline & $16(2+\varepsilon) \varepsilon^{4}$ & $16(2+\varepsilon) \varepsilon^{4}$ \\
\hline$\Lambda_{1}(\varepsilon \rightarrow 0)$ & $-\frac{1}{3}\left(1+\frac{\varepsilon}{2}\right)+O\left(\varepsilon^{2}\right)$ & $-\frac{1}{3}\left(1-\frac{\varepsilon}{2}\right)+O\left(\varepsilon^{2}\right)$ \\
\hline$\Lambda_{2}(\varepsilon \rightarrow 0)$ & $\frac{1}{8}\left(1+\frac{\varepsilon}{6}\right)+O\left(\varepsilon^{2}\right)$ & $\frac{1}{8}\left(1-\frac{7 \varepsilon}{6}\right)+O\left(\varepsilon^{2}\right)$ \\
\hline
\end{tabular}


Table 2: Convection-reaction dominated exit concentration for several kinetics.

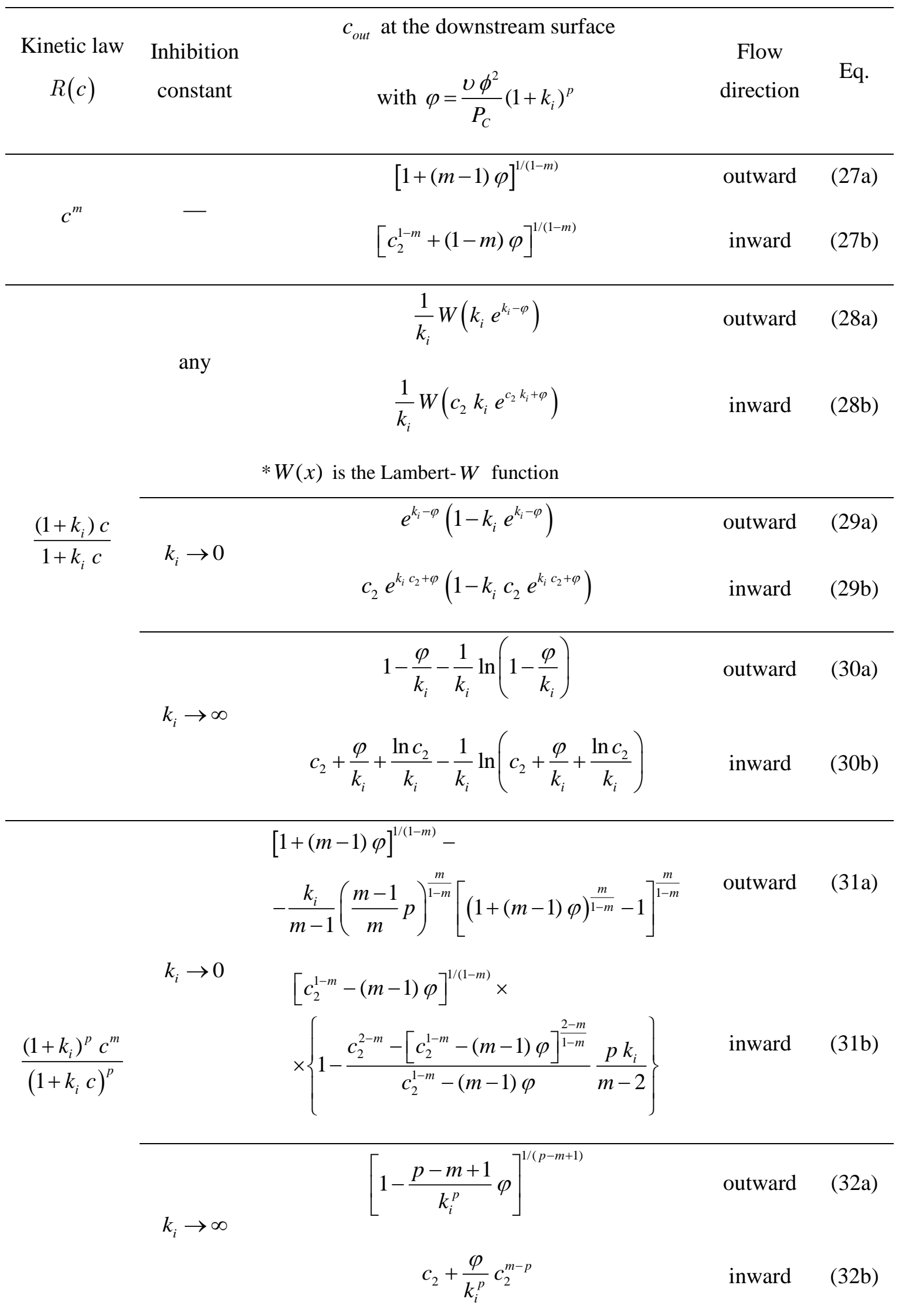


Table 3: Conditions for maximum effectiveness enhancement in Regime IV.

$R(c) \quad$ Optimum flow-through membrane Damköhler number $\varphi=\left(1+k_{i}\right)^{p} \frac{v \phi^{2}}{P_{C}} \quad$ Eq

Outward flow from [44]:

$\varphi=1.256+\frac{4.739}{v P_{C}}\left[(1+\varepsilon)^{2}-0.119-2 \varepsilon v\left(1-\frac{c_{2}}{4}\right)\right]$

Inward flow from [44]:

$\varphi=-1.256-\frac{1.161}{v P_{C}}\left[2 \varepsilon v\left(\frac{1}{c_{2}}-3.513\right)-3.595\right]$

Outward flow: $\varphi \simeq 1+\varphi_{0}+\frac{\varphi_{1}}{P_{C}}$

$\varphi_{0}=\frac{2+m-m^{m /(m-1)}}{3+m-m^{m /(m-1)}}$

$\varphi_{1}=\frac{1-(1+\varepsilon)^{2} m^{m /(1-m)}-\left(1-c_{2}\right) \varepsilon v}{(3+m) m^{m /(1-m)} v-v}$

$c^{m}$

Inward flow: $\varphi_{2}=\frac{v}{-P_{C}} \frac{t_{m}^{2}}{D_{\text {eff }}} \hat{c}_{2}^{m-1}=1+\varphi_{0}+\frac{\varphi_{1}}{-P_{C}}$

$\varphi_{0}=\frac{(2+m) m^{1 /(m-1)}-m}{(5+2 m) m^{1 /(m-1)}-2 m}$

$\varphi_{1}=\frac{1+m^{m /(1-m)}+\left(3-1 / c_{2}\right) v \varepsilon}{(5+2 m) m^{m /(1-m)} v-2 v}$

$\frac{\left(1+k_{i}\right) c}{1+k_{i} c} \quad|\varphi|=4-e+k_{i} c_{i n}\left(1+\frac{0.5}{1+k_{i}^{2}}\right)$ 

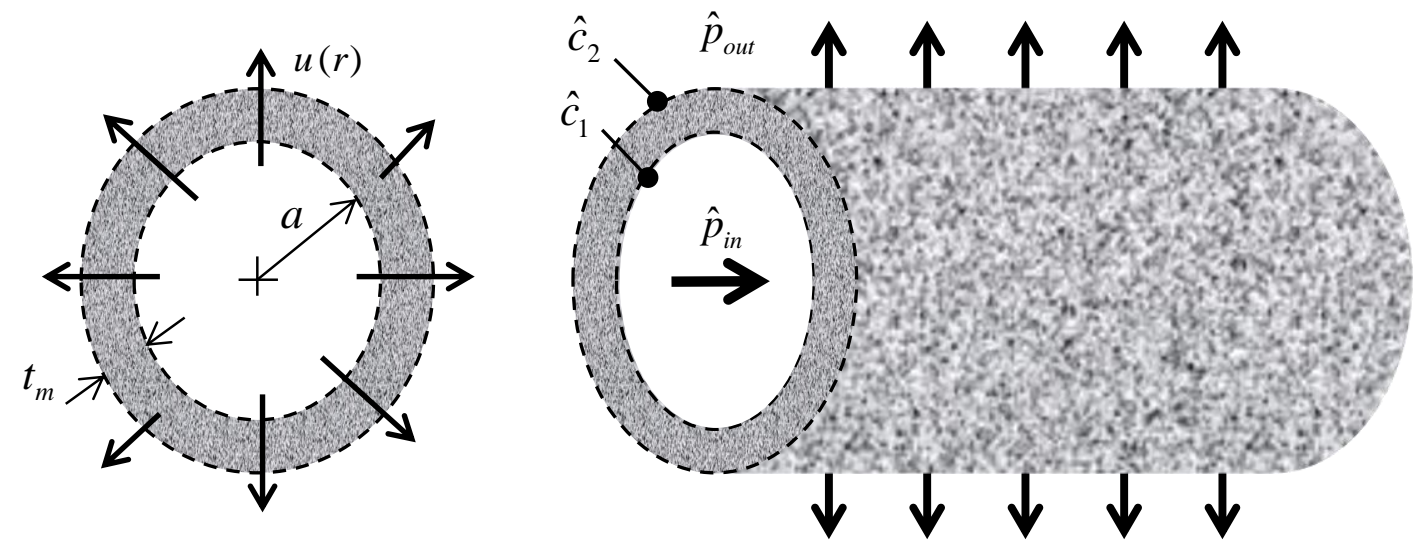

Fig. 1

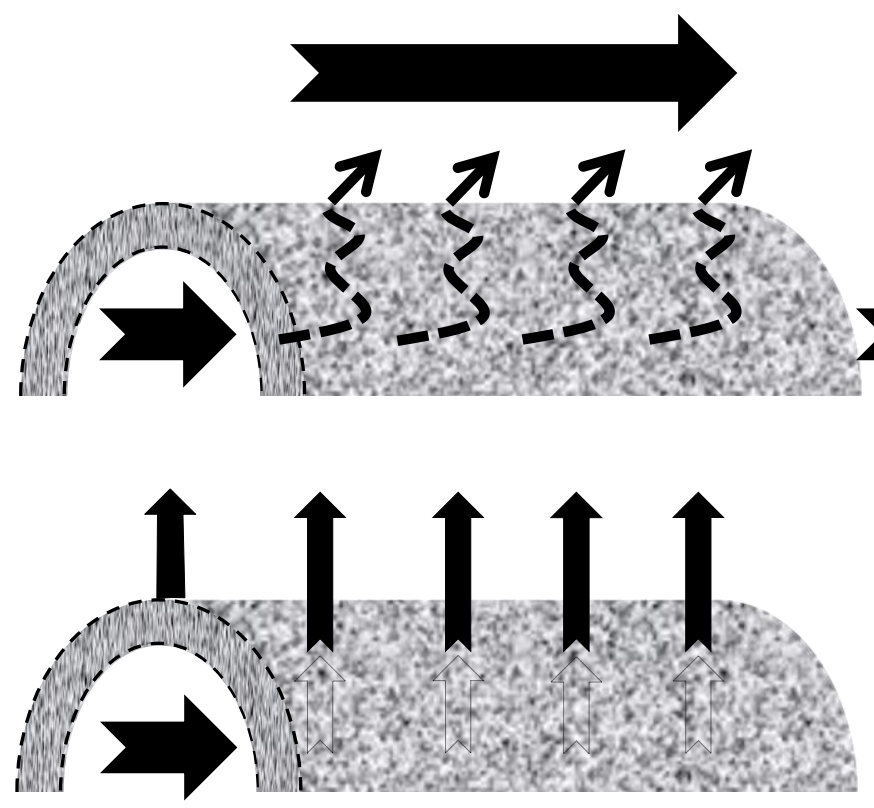

(A)

Fig. 2

(B) 


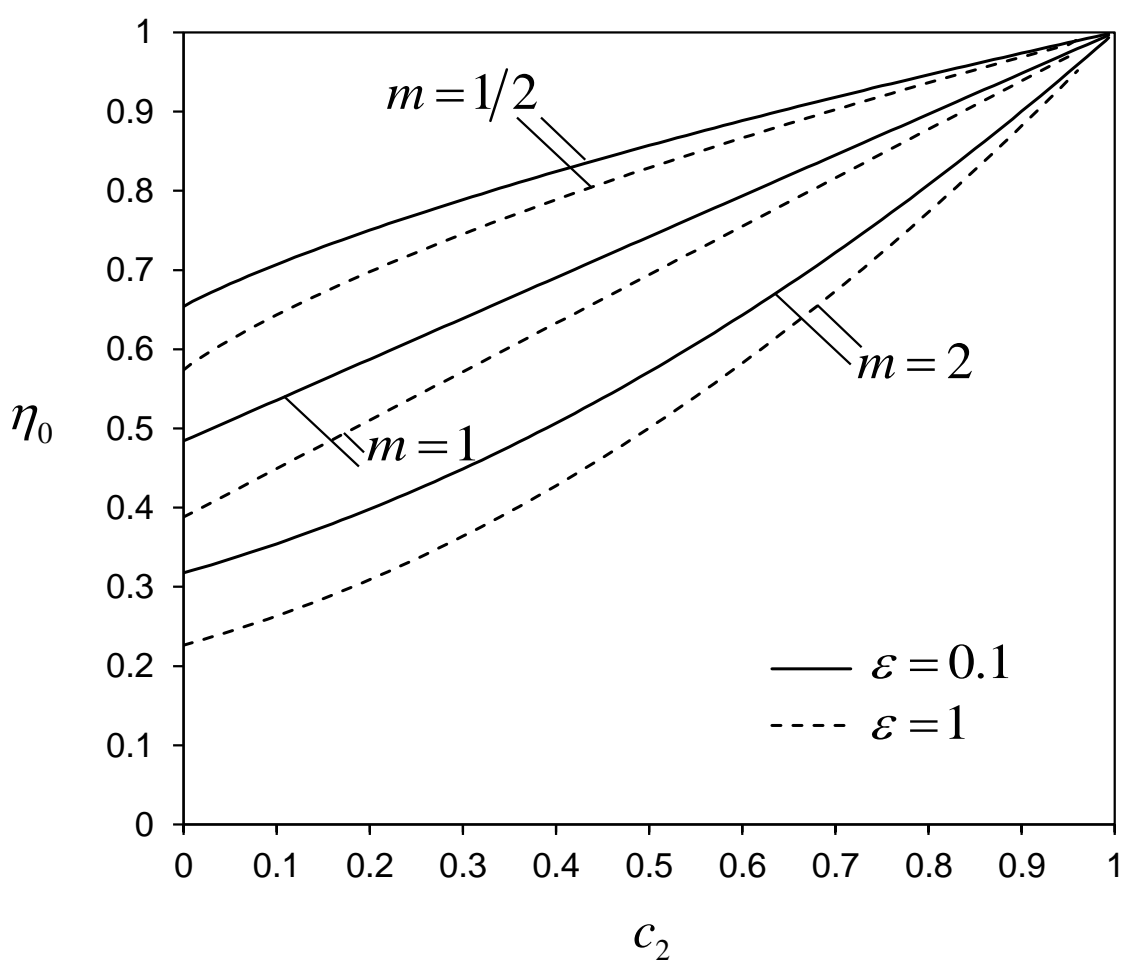

Fig. 3

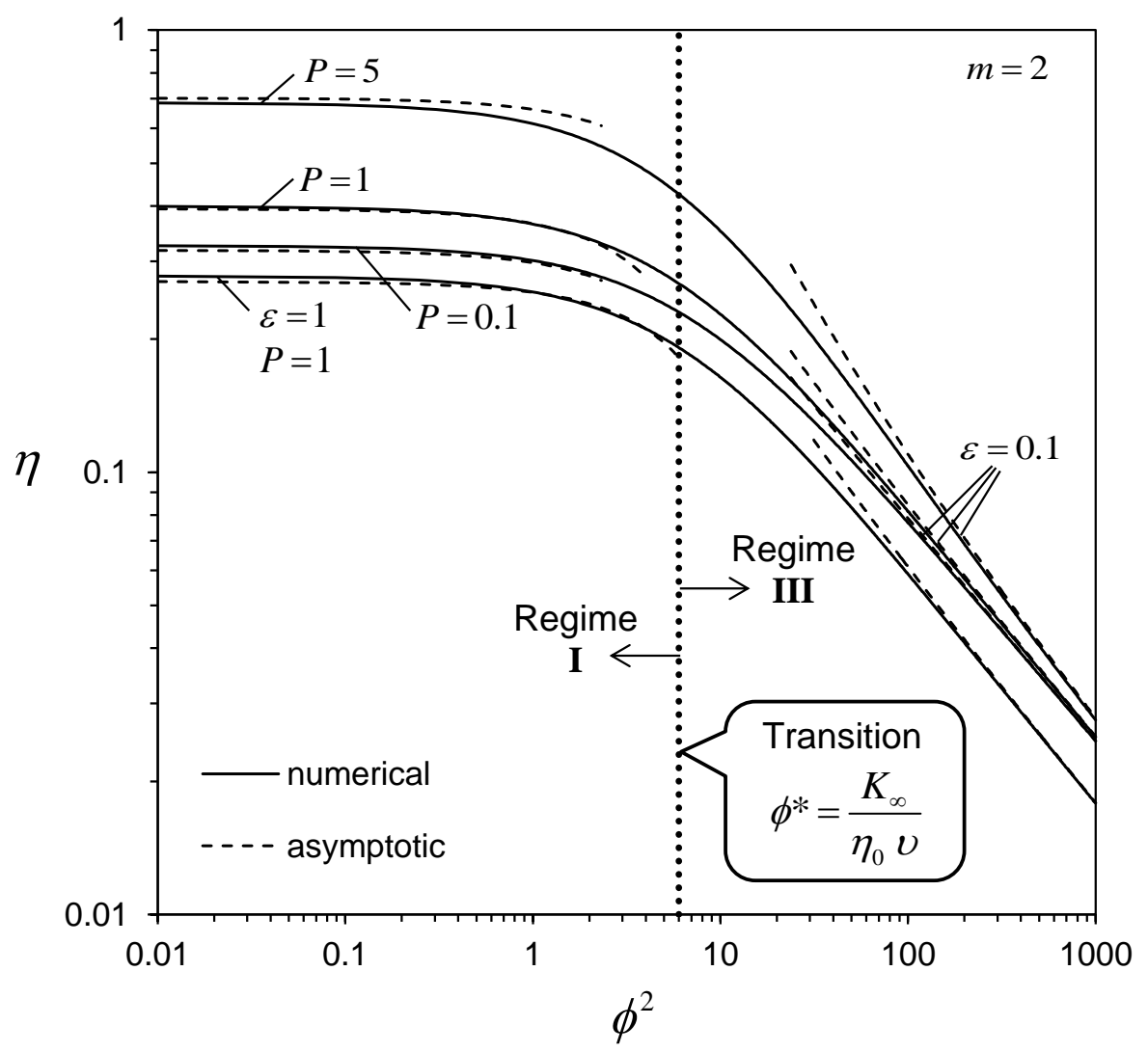

Fig. 4 


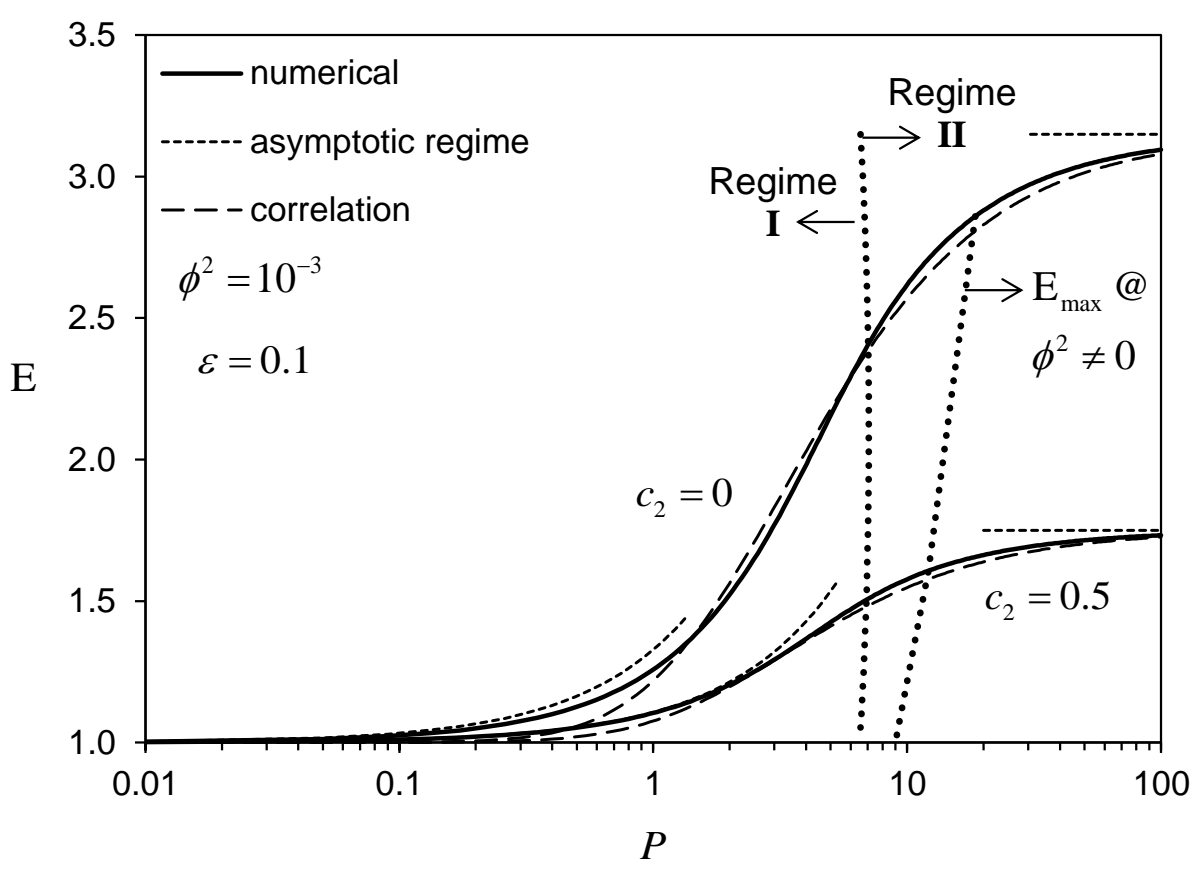

Fig. 5 (a)

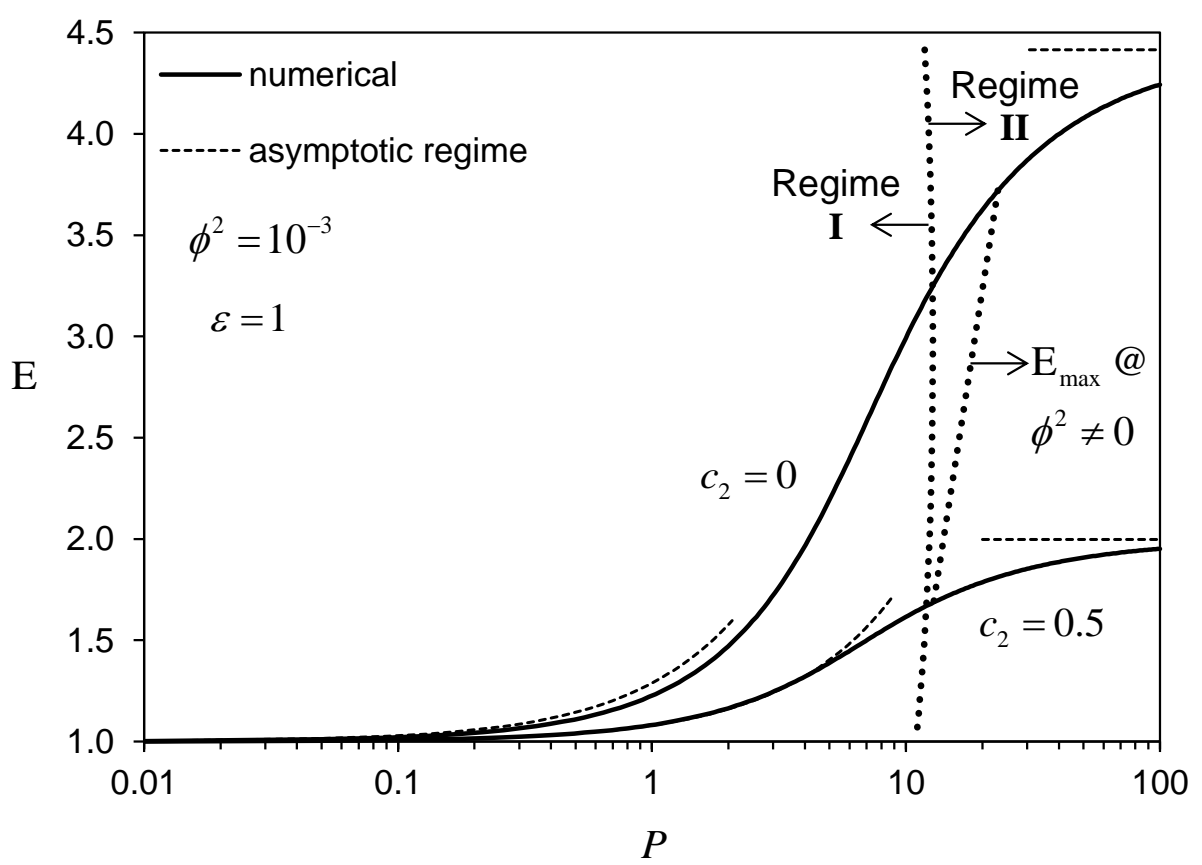

Fig. 5(b) 


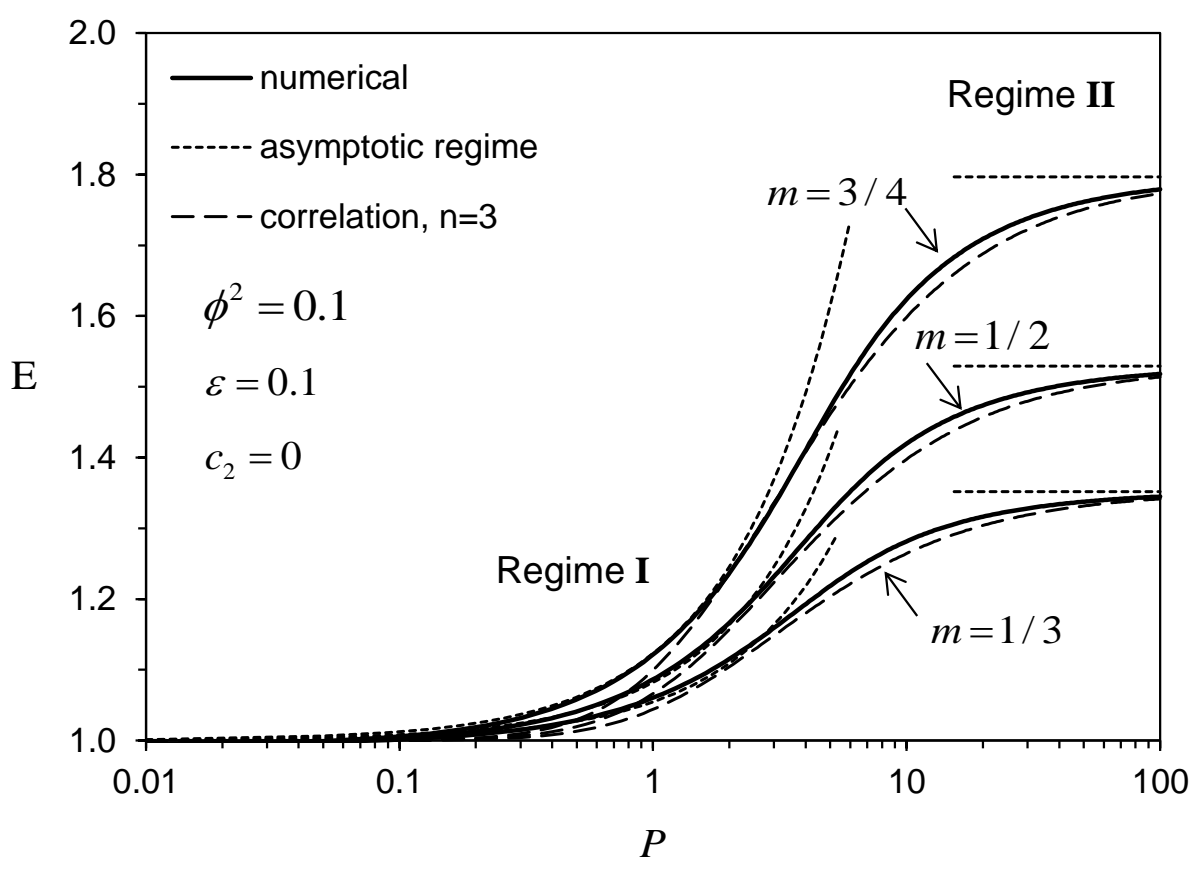

Fig. 5 (c)

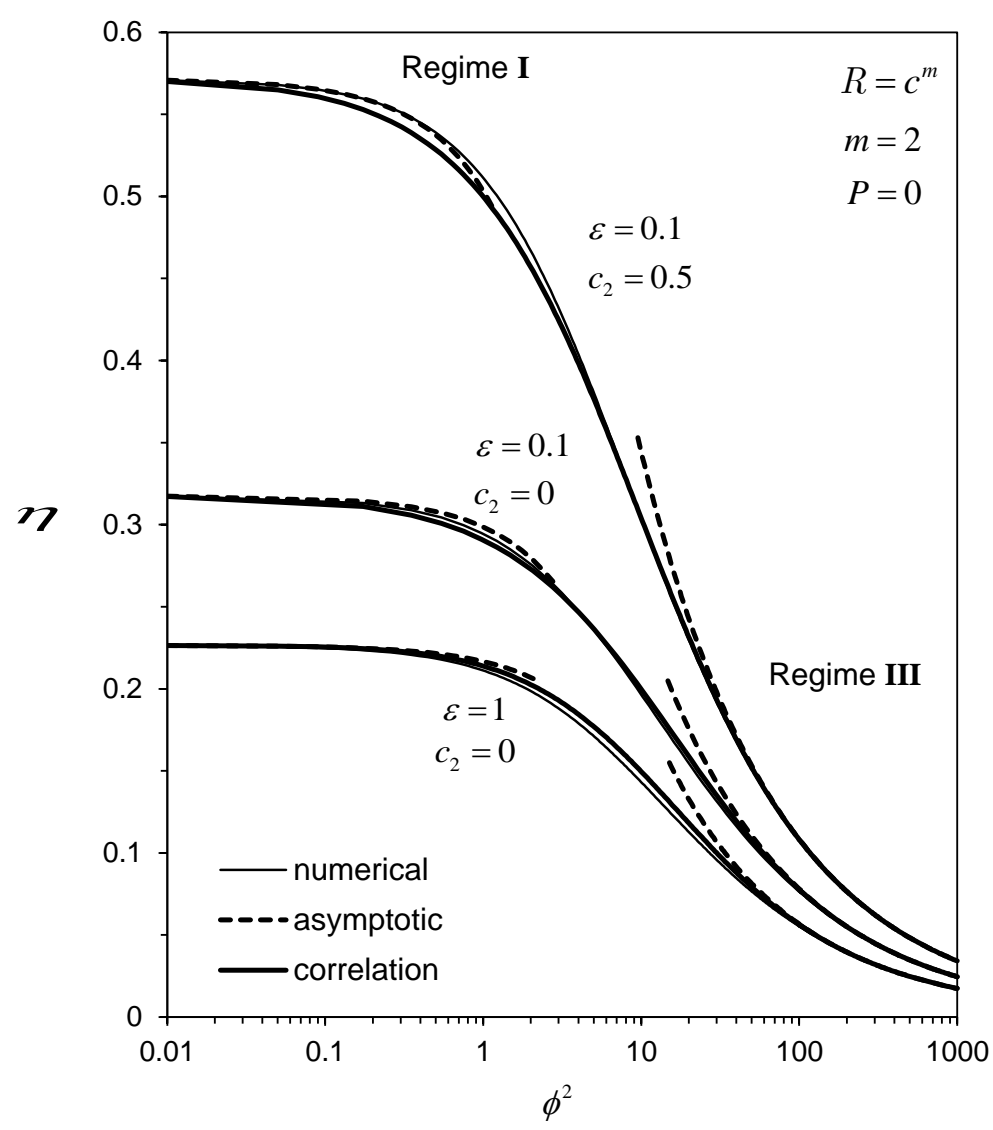

Fig. 6 


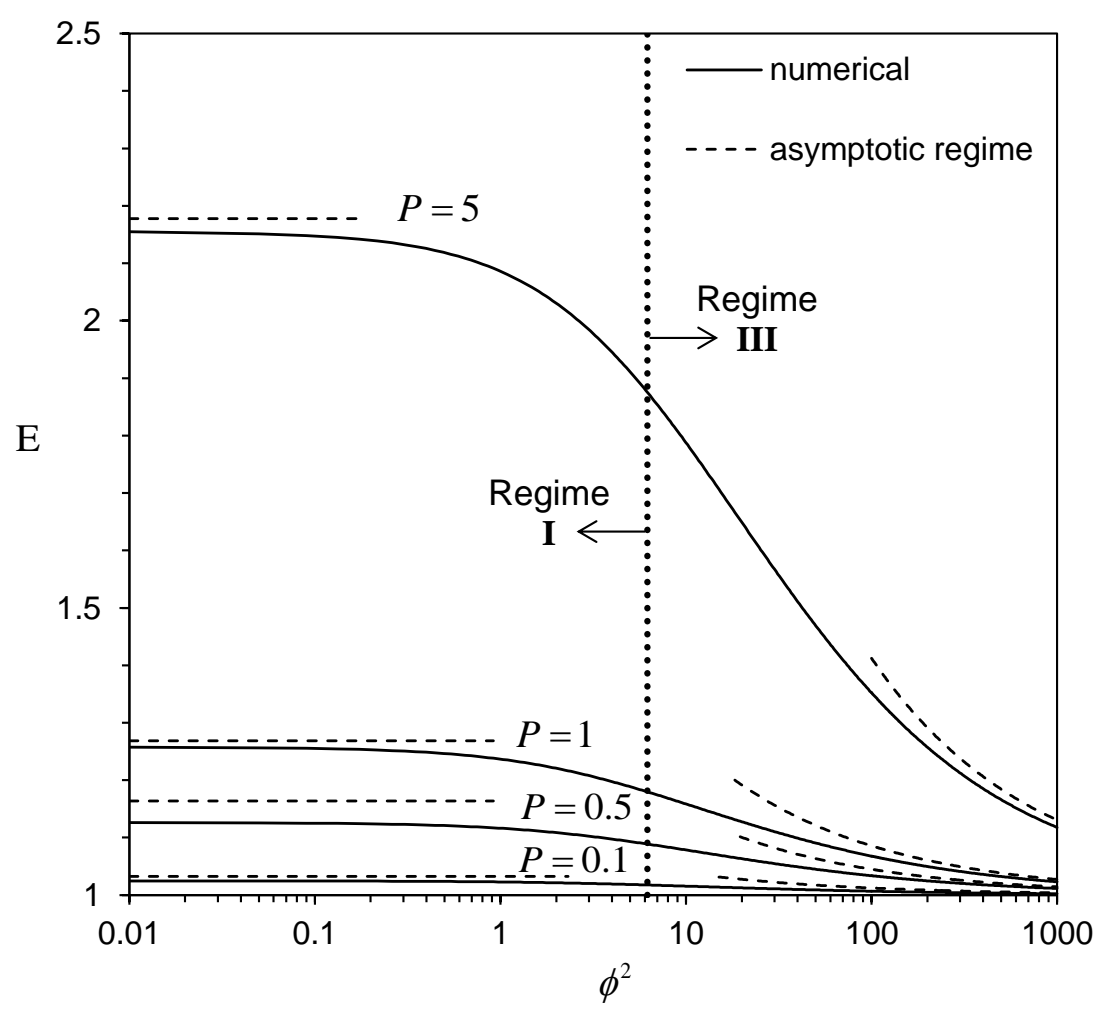

Fig. 7

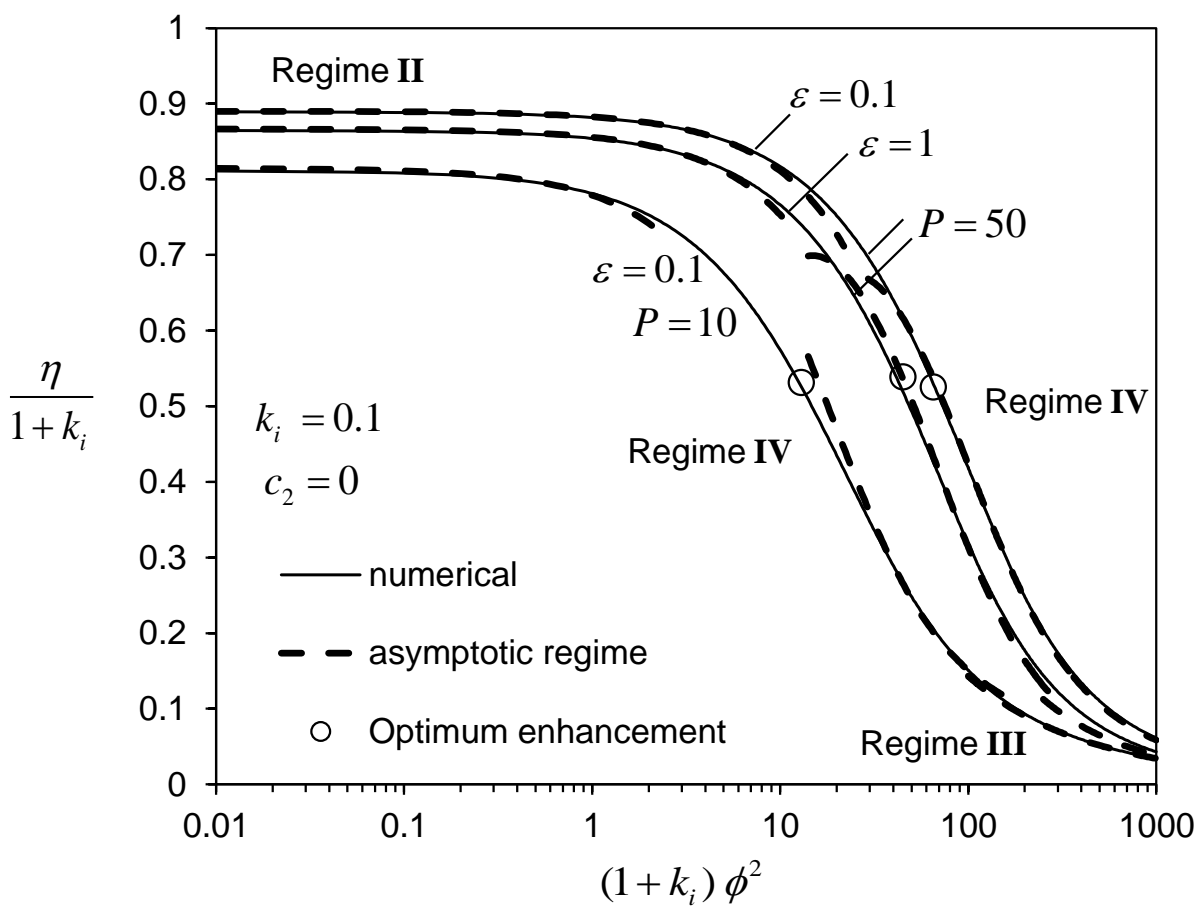

Fig. 8 (a) 


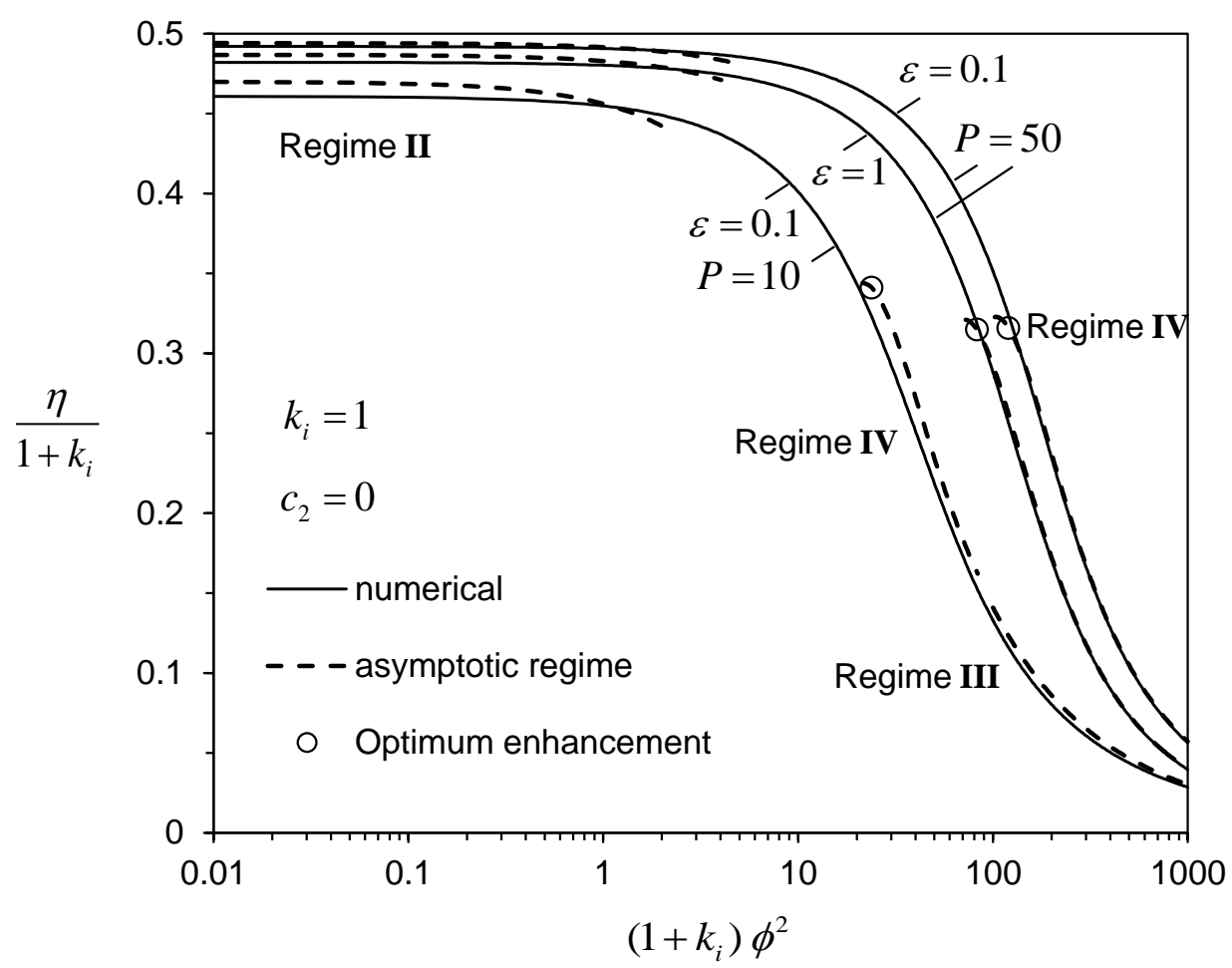

Fig. 8 (b)

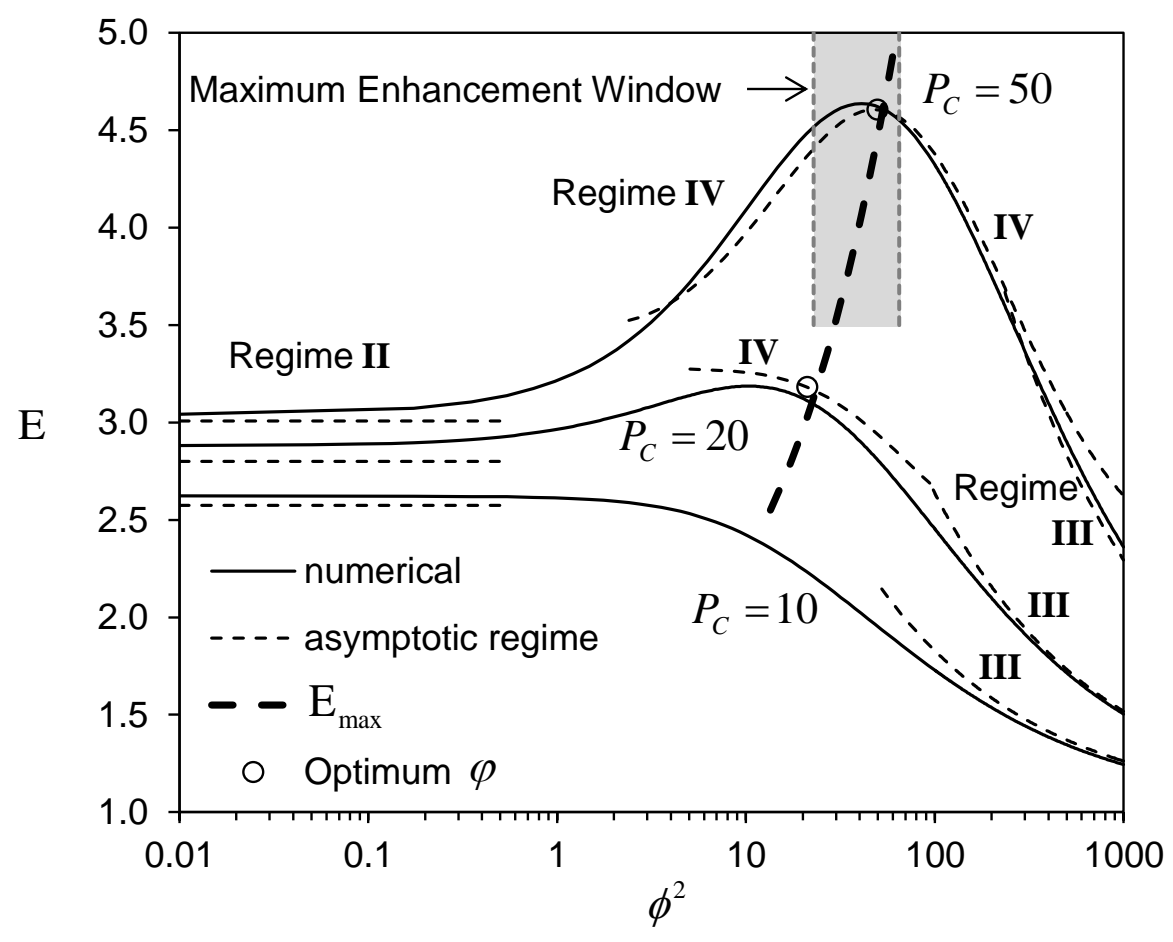

Fig. 9 


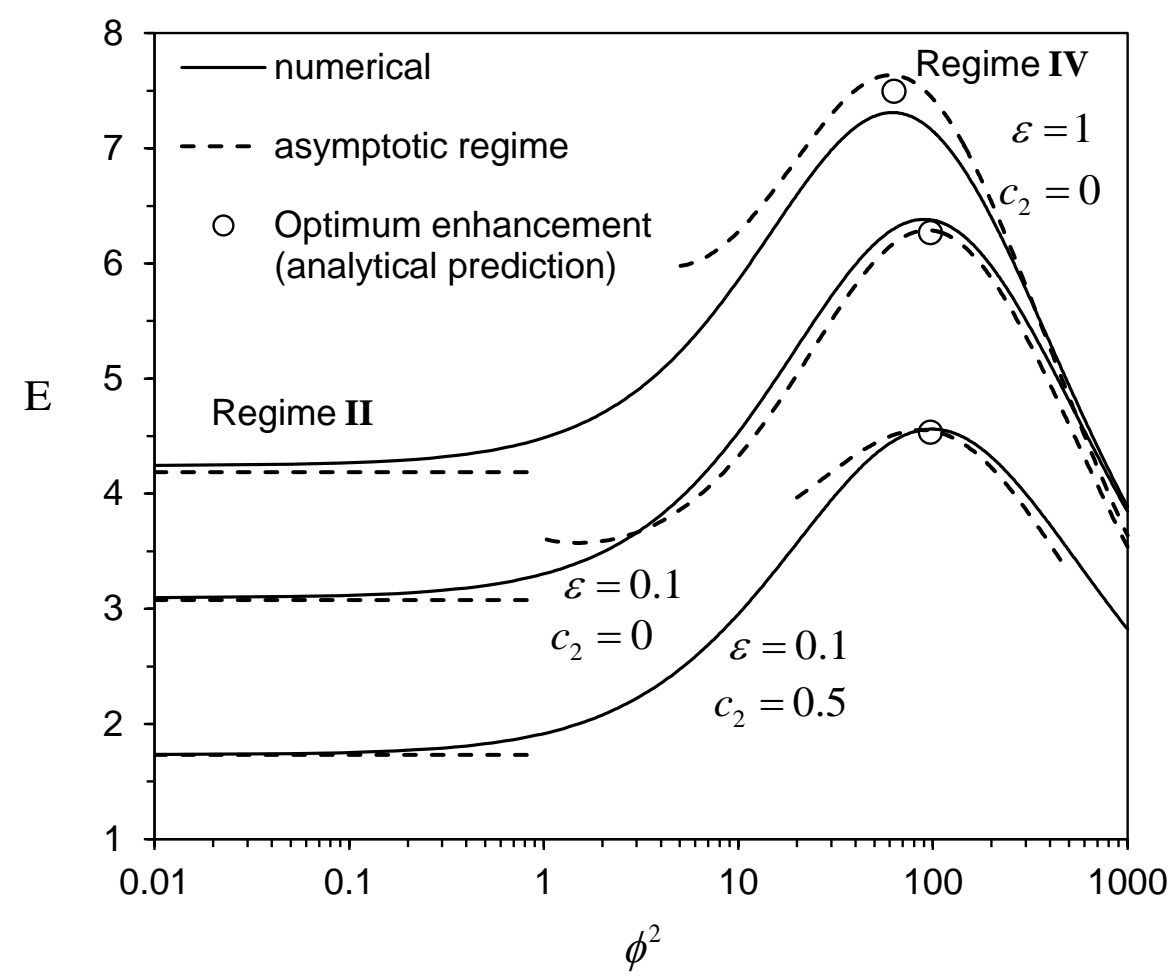

Fig. 10

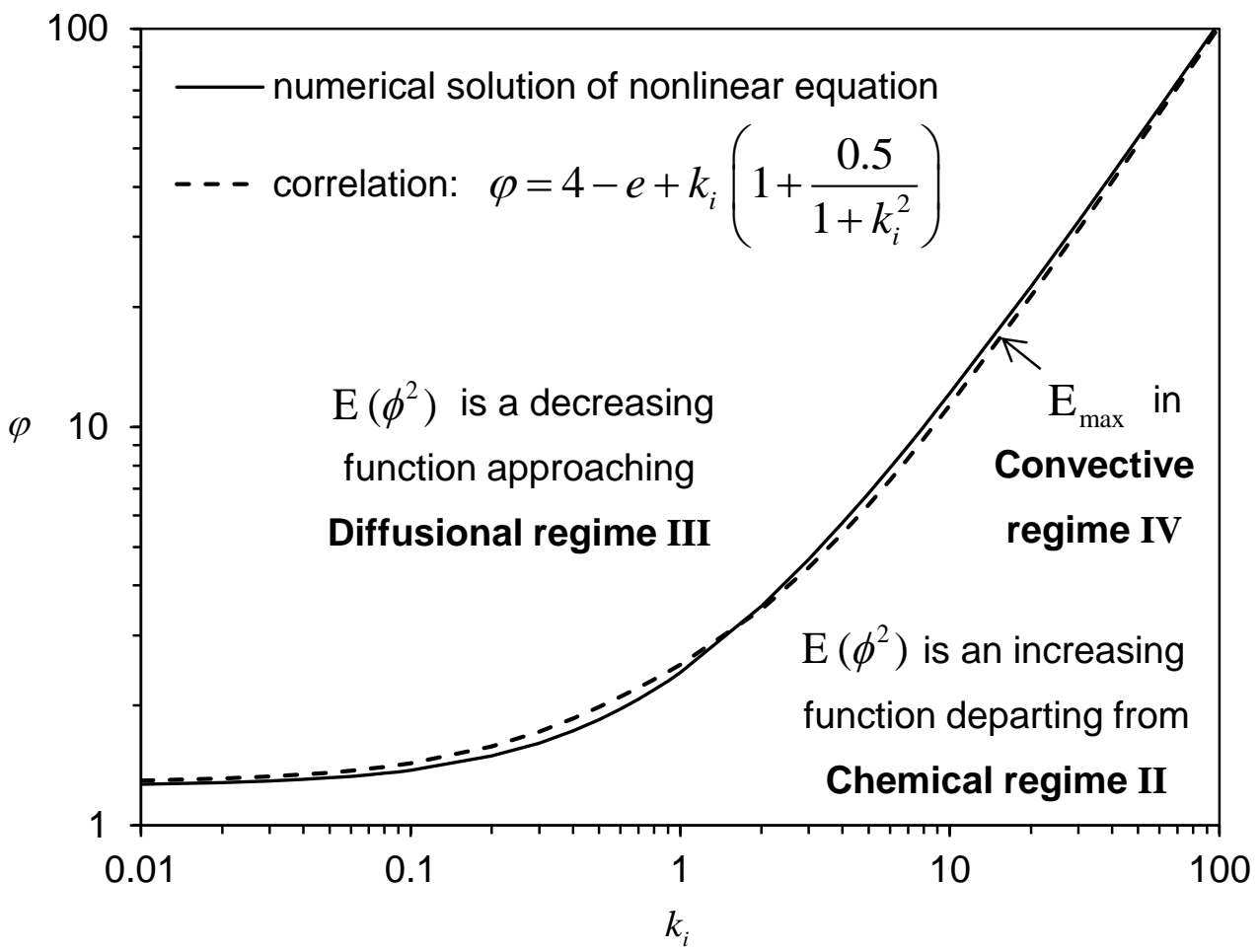

Fig. 11 


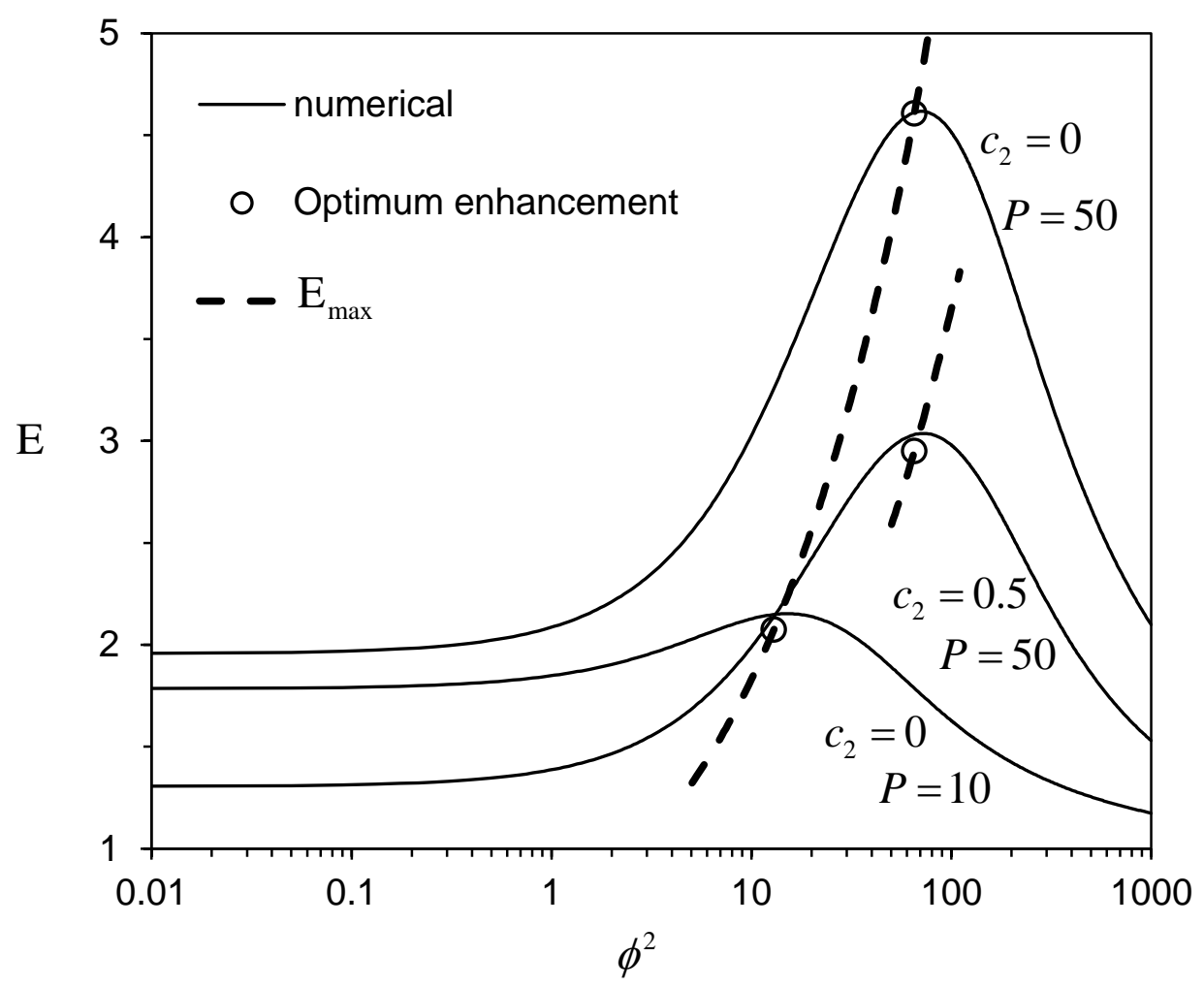

Fig. 12

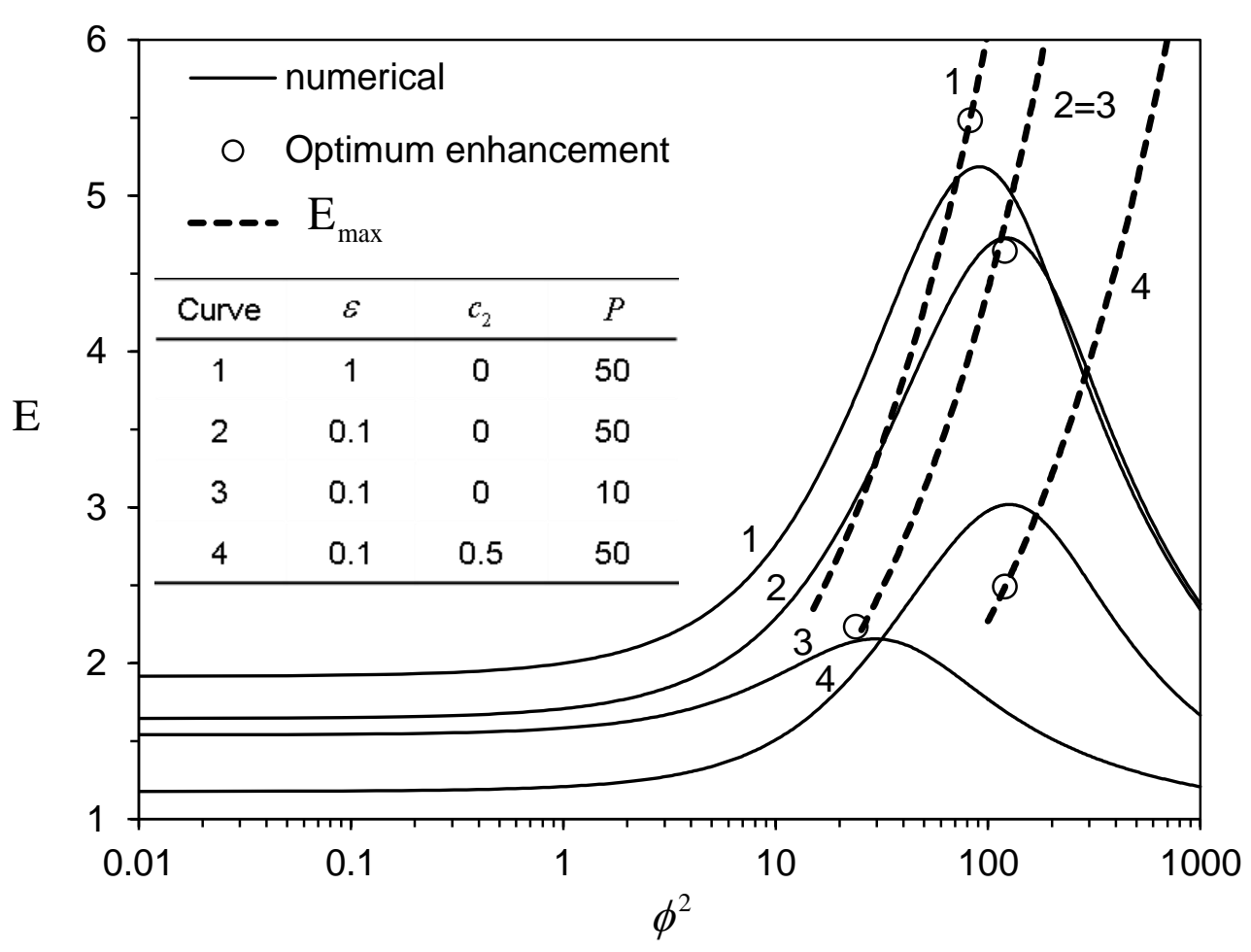

Fig. 13 


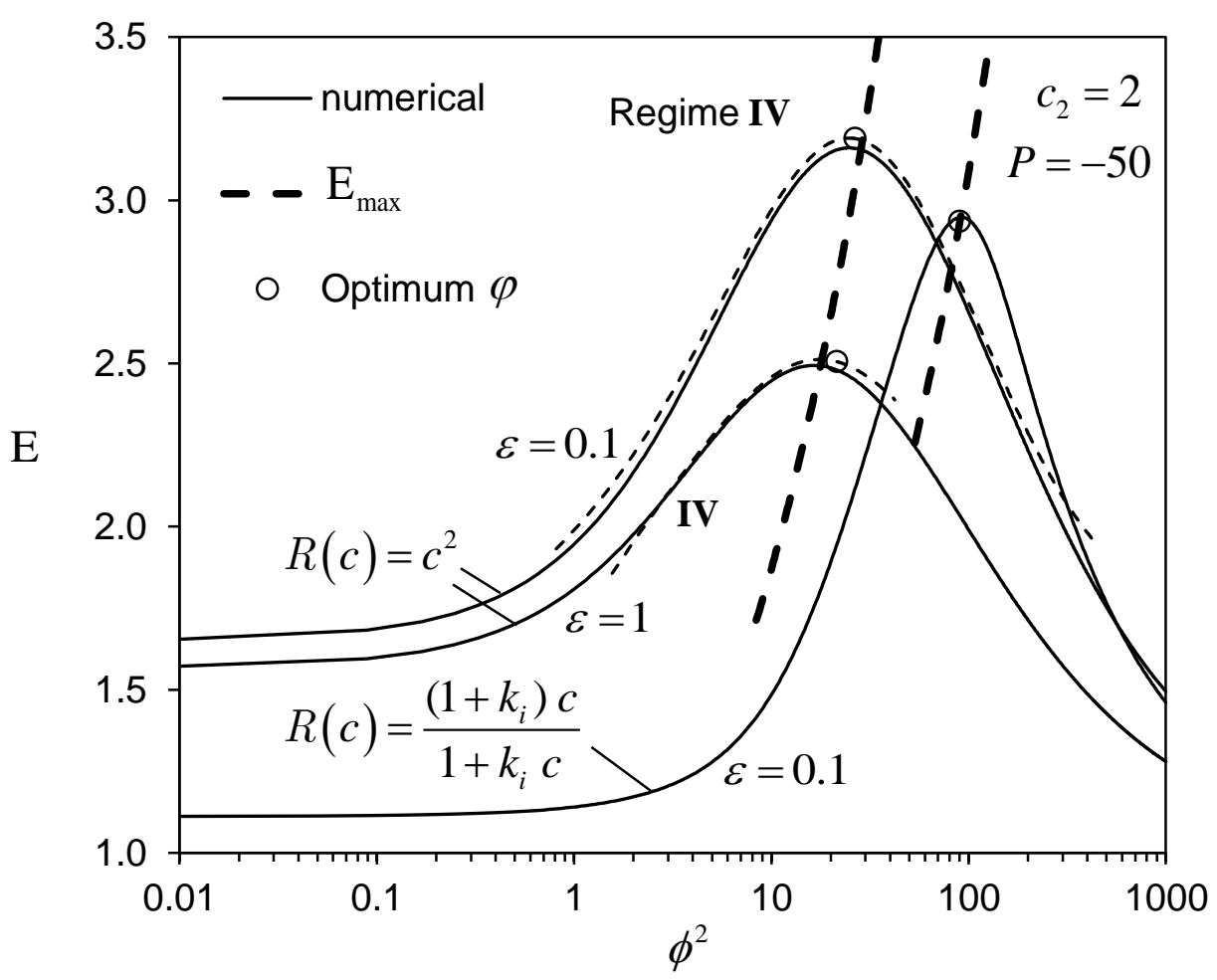

Fig. 14

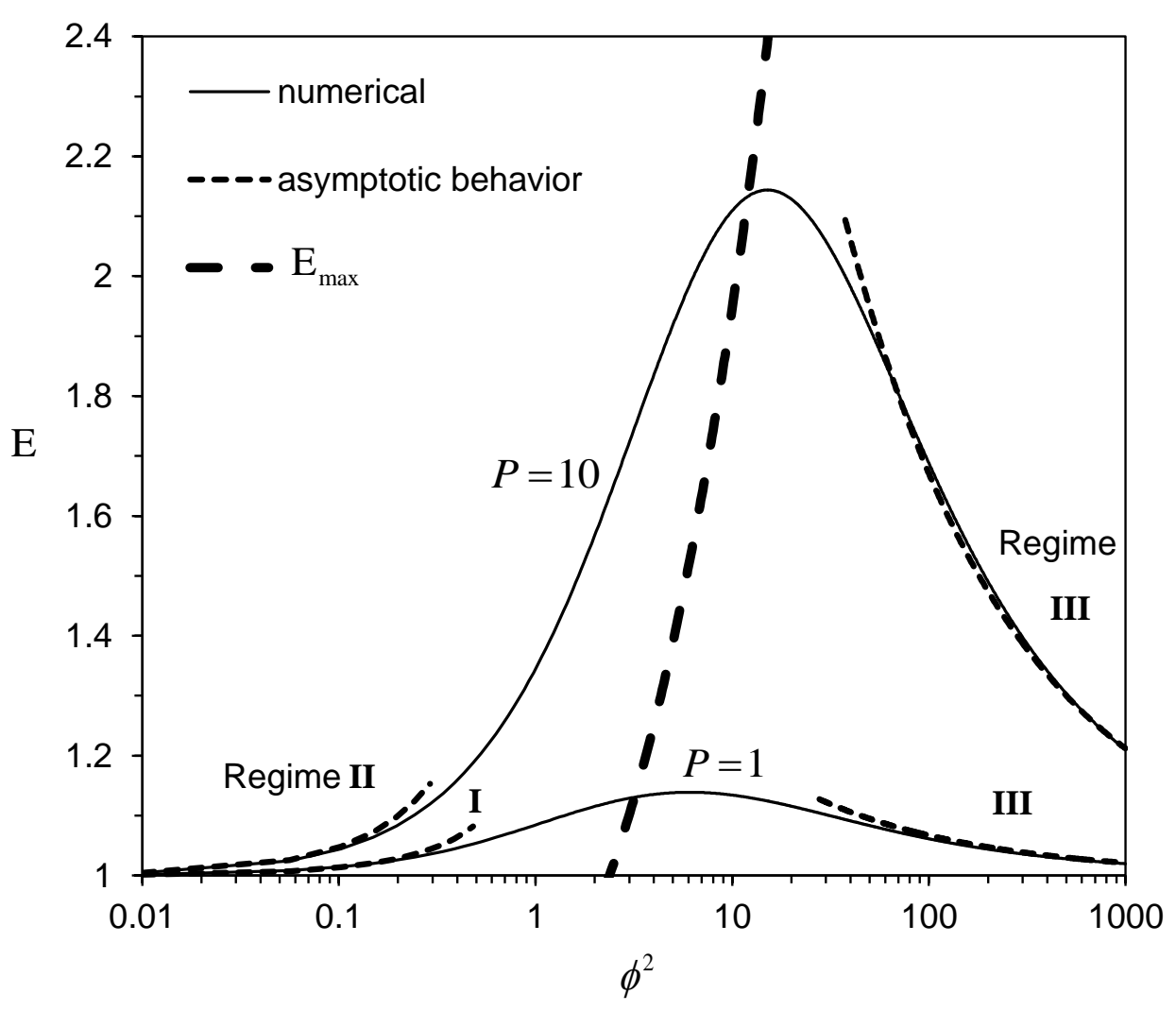

Fig. 15 (a) 


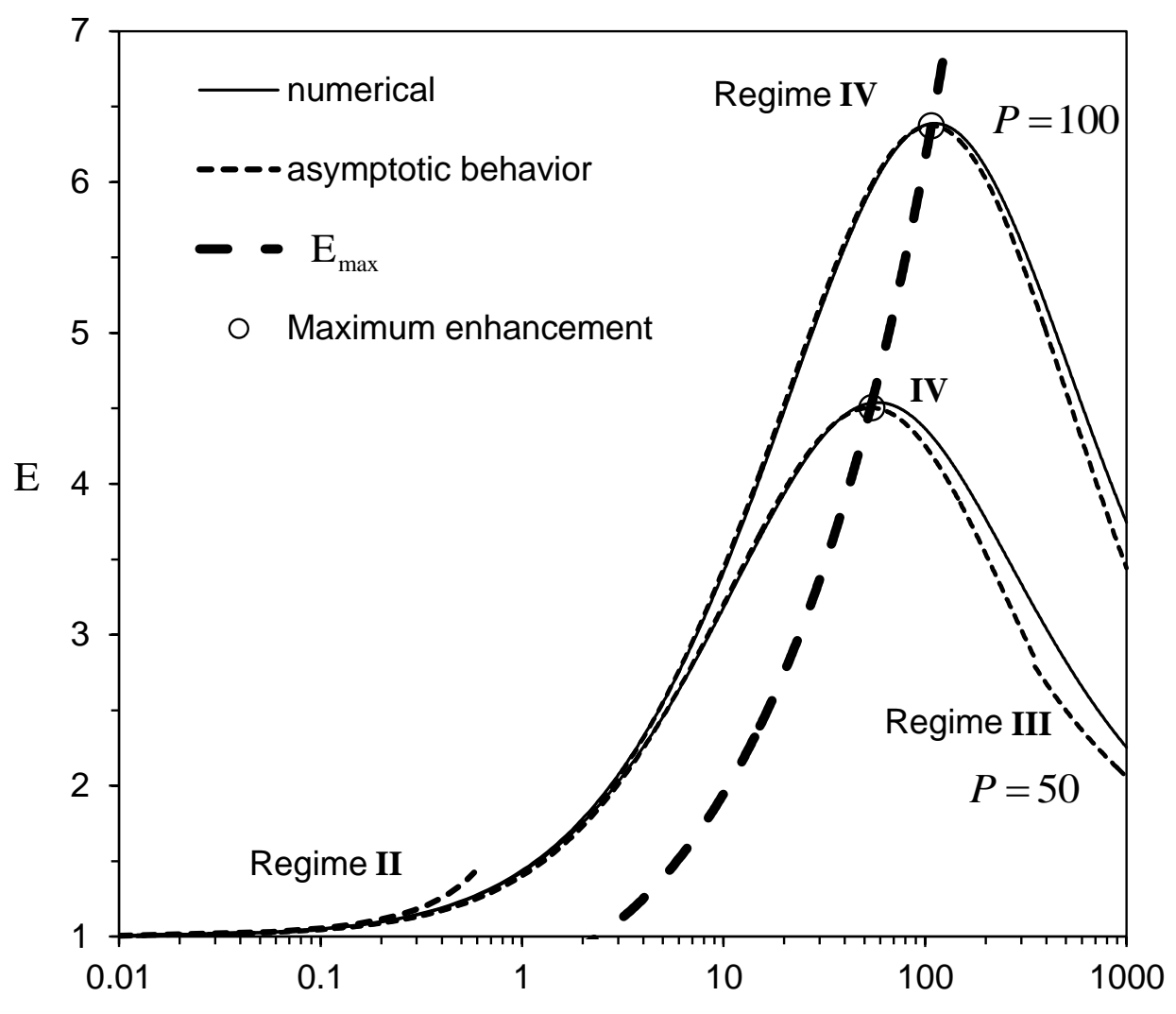

Fig. 15 (b)

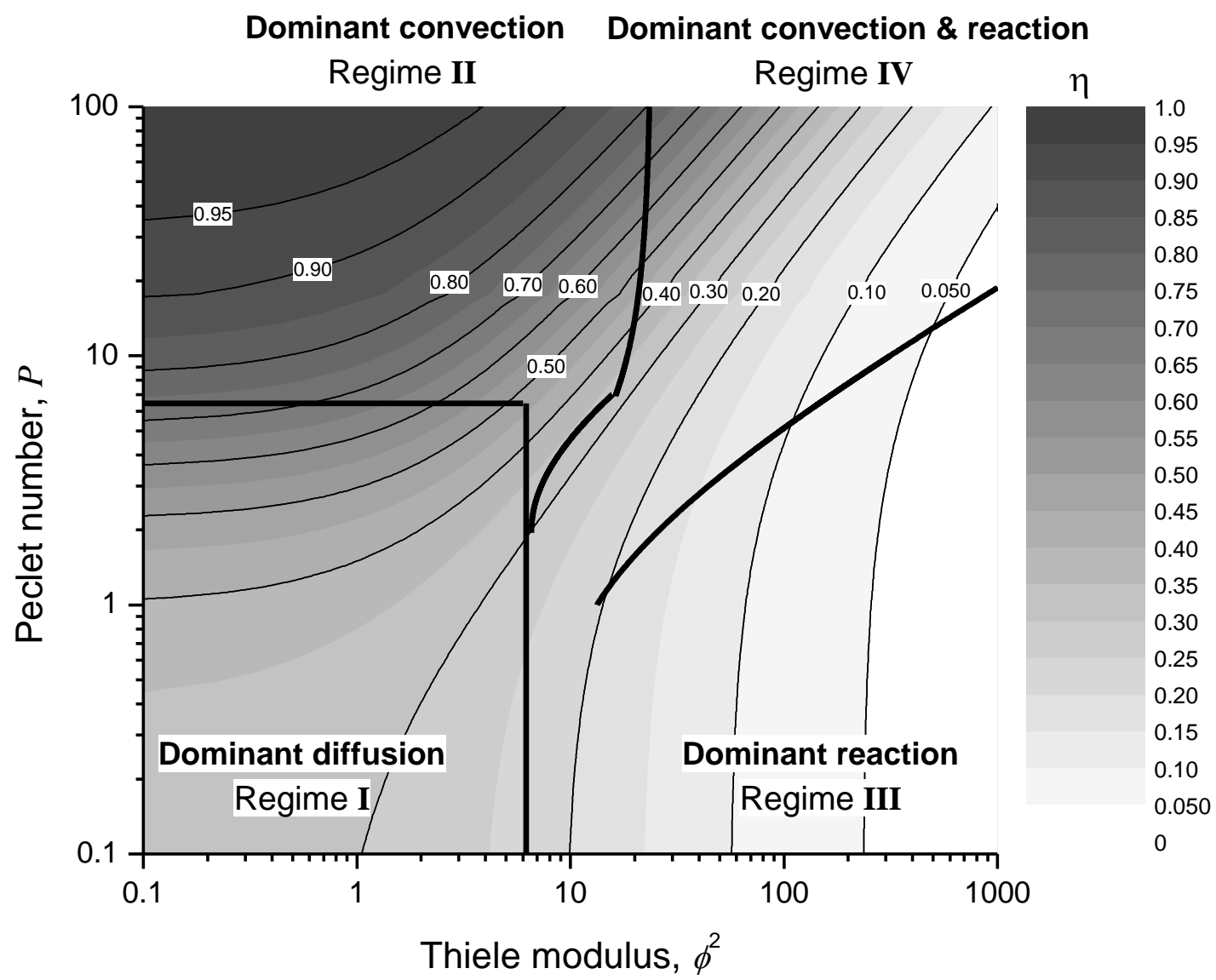

Fig. 16 


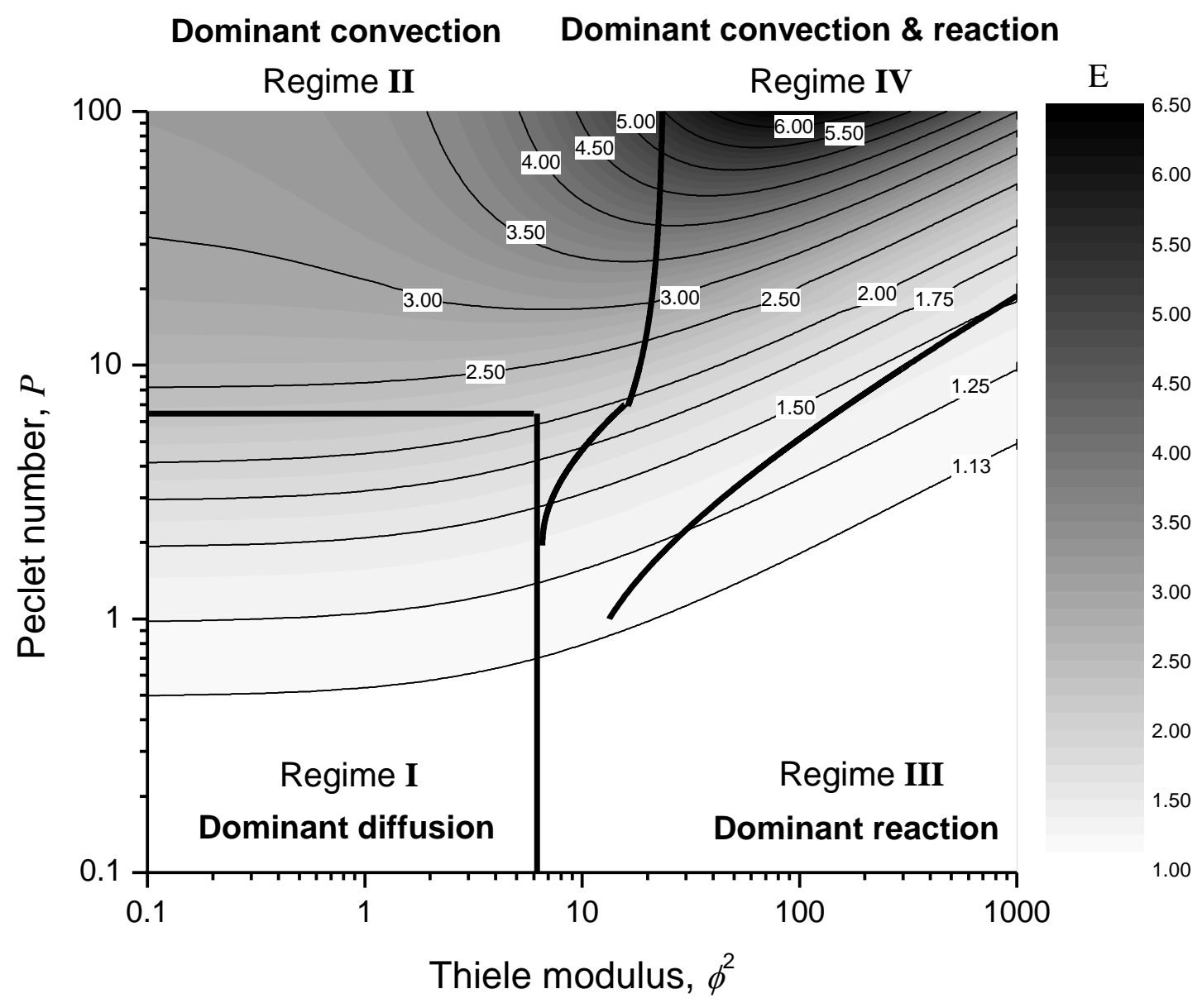

Fig. 17 


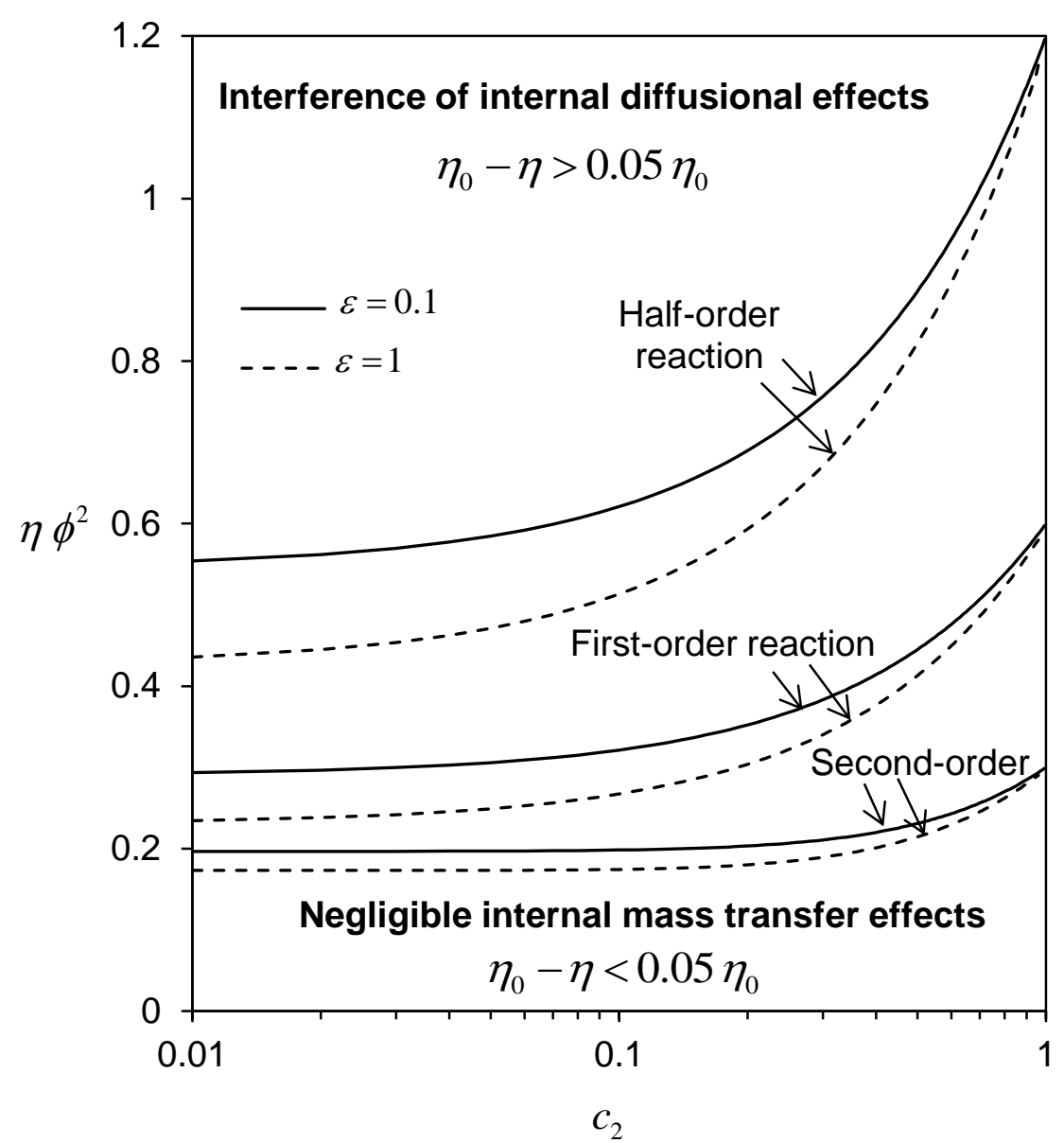

Fig. A.1 